\title{
Integrated Fault-tolerant Control for Close Formation Flight
}

\author{
Chun Liu, Bin Jiang, Senior Member, IEEE, Ron J. Patton, Life Fellow, IEEE, \\ and Ke Zhang, Senior Member, IEEE
}

\begin{abstract}
This study investigates the position-tracking and attitude-tracking control problem of close formation flight with vortex effects under simultaneous actuator and sensor faults. On the basis of the estimated state and fault information from unknown input observers and relative output information from neighbors, an integration of decentralized faultestimation and distributed fault-tolerant control is developed to deal with bidirectional interactions and to guarantee the asymptotic stability and $H_{\infty}$ performance of close formations.
\end{abstract}

\section{Index Terms}

Decentralized fault estimation, distributed fault-tolerant control, bidirectional interactions, actuator and sensor faults, close formation flight.

\section{INTRODUCTION}

Formation control of unmanned aerial vehicles (UAVs) has gained considerable attention in recent years. Close formation flight $(\mathrm{CFF})$ is defined as formation geometry with a lateral spacing that is less than a wingspan in between UAVs. Multiple UAVs that fly in a CFF pattern can achieve a significant reduction in power demand, thereby improving cruise performances, extending mileage and increasing payload via induced drag reduction[1], [2]. This drag reduction in CFF is due to beneficial wake-vortex encounters. A Lead UAV generates vortices that induce an up-wash on the wing and a side-wash on the vertical tail behind the Wing UAVs[3], [4]. Thus, positive effects can be obtained by specifying the close formation concept.

Various control approaches, such as adaptive control[5], sliding-mode control[6] and receding horizon control[7], have been introduced by studies that focused on CFF problems. Most of the results are based on CFF models that

C. Liu, B. Jiang and K. Zhang are with the College of Automation Engineering, Nanjing University of Aeronautics and Astronautics, Nanjing 210016, China (e-mail: binjiang@ @uaa.edu.cn).

R. J. Patton is with the School of Engineering and Computer Science, University of Hull, Cottingham Road, Hull HU6 7RX, UK.

This work was supported by National Natural Science Foundation of China (61773201, 61622304 and 61673207); Fundamental Research Funds for the Central Universities (NE2014202); Priority Academic Program Development of Jiangsu Higher Education Institutions; Qing Lan Project of Jiangsu Province; Postgraduate Research and Practice Innovation Program of Jiangsu Province (KYCX17_0269); and China Scholarship Council (201706830025).

(C) 2020 IEEE. Personal use of this material is permitted. Permission from IEEE must be obtained for all other uses, in any current or future media, including reprinting/republishing this material for advertising or promotional purposes, creating new collective works, for resale or redistribution to servers or lists, or reuse of any copyrighted component of this work in other works. 
either linearize nonlinear dynamics or disregard the vortex effects. Additional aerodynamic coupling effects, sixdimensional CFF equations and proportional-integral formation-hold autopilots were established[8]. The literature indicates that research on nonlinear CFF has received little attention thus far. A decentralized robust control strategy was presented by using a high-gain observer for nonlinear CFF[9]. In addition, CFF problems only focused on the separated position-tracking[2], [10] or attitude-tracking issues[11]. Tracking control of roll dynamics in CFF was proposed by using $H_{\infty}$ techniques to withstand lateral aerodynamical perturbations[11].

However, faults may occur in one or more UAVs. Such faults can cause undesirable performances or even lead to catastrophic results in CFF systems. Therefore, fault-tolerant formation control (FTFC) is required to guarantee the stability and satisfactory properties of CFF systems. Three fault types, namely, communication[12], [13], actuator[14]-[19] and sensor faults[20], [21], can be considered for CFF. An FTFC design for the formation flight of multiple UAVs was proposed to accommodate actuator faults on the basis of reference generator and finite-time convergence target[15]. Permanent and intermittent actuator faults were also dealt with by developing an FTFC scheme for UAV formation control[16]. On the one hand, most FTFC schemes in the literature are applied to counteract separated communication, actuator or sensor faults, and the problem of coping with simultaneous actuator and sensor faults in UAV formation flight should be investigated. On the other hand, aside from the fact that previous works[15],[22]-[26] have only focused on separated fault estimation (FE) and fault-tolerant control (FTC) protocols, the presented control schemes have not considered the bidirectional interactions between FE and FTC systems and the direct application of estimated fault information from FE to FTC systems. Lan and Patton[27], [28] proposed integrated FE and FTC protocols for uncertain systems in the presence of Lipschitz nonlinearities, disturbances and simultaneous actuator and sensor faults. That work simultaneously handled the effects of mutual couplings from the disturbances and nonlinearities between FE and FTC systems. Furthermore, their approaches[27], [28] used the so-called integrated FE/FTC design, which is known as a decentralized structure[20]. This integrated FE/FTC design cannot be applied in distributed formation control due to mechanical interconnections and restricted topology problem in practical applications. The distributed control designs[14], [17], [19],[29]-[32] were equipped with information interactions from their coupled neighbors and had the advantages of low cost and easy implementation. The consensus formation protocol in [17] only required the neighbors' relative information, whereas the FTC protocol in [19] needed both its own information and the information of its neighbors to construct a local FTFC approach in a fully distributed fashion. A distributed FTC strategy was previously proposed on the basis of adaptive approximations and local-state information for nonlinear uncertain interconnected systems[29]. Therefore, how to develop an integrated FE and FTC design with bidirectional interactions in a distributed fashion for CFF systems, in the presence of simultaneous actuator and sensor faults, is a challenging topic.

The major contributions of this study can be summarized as follows. (i) The nonlinear CFF modeling with vortex effects and simultaneous actuator and sensor fault modeling are proposed. Feasible reference models in [15], [24] are not required, instead, a strategic $H_{\infty}$ design is needed in CFF systems. (ii) The proposed unknown input observers in the decentralized FE system are developed to estimate the faults and states without prior information requirements on the basis of previous works[27], [28]. The FE design does not require the bounds of the parametric system uncertainties[17], [24] and faults[32] nor any global knowledge about communication 
topology[17]. (iii) This study considers the integration of decentralized FE and distributed FTC protocols compared with the globally decentralized FE/FTC structure[27], [28]. The bidirectional interactions between FE and FTC systems are also considered. Furthermore, unlike FTC designs based on local state information[29] or estimated fault information[17], [24], [31], the proposed FTC protocol is implemented in a fully distributed manner based on the estimated information in the FE system and on the output information of the neighbors.

The remainder of this paper is organized as follows: in Section II, the model description and system formulation are introduced. Section III is devoted to the decentralized FE design. Two types of distributed control schemes including the separated and integrated FE/FTC designs are presented in Section IV to achieve the good tracking of attitude and position commands. Simulation in Section V validates the efficiency of the proposed control algorithm. Finally, conclusions follow in Section VI.

Notations: The symbol $\dagger$ denotes the pseudo inverse, $\otimes$ denotes the kronecker product, $\operatorname{He}(X)=X+X^{T}$, and $\star$ represents the symmetric part of the specific matrix.

Graph theory: An undirected graph $\mathcal{G}$ is a pair $(\nu, \varsigma)$, where $\nu=\left\{\nu_{1}, \cdots, \nu_{N}\right\}$ is a nonempty finite set of nodes and $\varsigma \subseteq \nu \times \nu$ is a set of edges. The edge $\left(\nu_{i}, \nu_{j}\right)$ is denoted as a pair of distinct nodes $(i, j)$. A graph is said to be undirected with the property $\left(\nu_{i}, \nu_{j}\right) \in \varsigma$ that signifies $\left(\nu_{j}, \nu_{i}\right)$ for any $\nu_{i}, \nu_{j} \in \nu$. Node $j$ is called a neighbor of node $i$ if $\left(\nu_{i}, \nu_{j}\right) \in \varsigma$. The set of neighbors of node $i$ is denoted as $\mathcal{N}_{i}=\left\{j \mid\left(\nu_{i}, \nu_{j}\right) \in \varsigma\right\}$. The adjacency matrix $\mathcal{A}=\left[a_{i j}\right]_{N \times N}$ is represented as the graph topology. $a_{i j}$ is the weight coefficient of the edge $\left(\nu_{i}, \nu_{j}\right)$ and $a_{i i}=0, a_{i j}=1$ if $\left(\nu_{i}, \nu_{j}\right) \in \varsigma$, otherwise $a_{i j}=0$. The Laplacian matrix $\mathcal{L}=\left[l_{i j}\right]_{N \times N}$ is defined as $l_{i j}=\sum_{i \neq j} a_{i j}$ and $l_{i j}=-a_{i j}, i \neq j$.

\section{MOdEL DESCRIPTION AND SYSTEM FORMULATION}

In this section, the CFF modeling including formation-hold autopilots, kinematics, and aerodynamic coupling vortex effects are effectively established. The simultaneous actuator and sensor fault modeling in the longitudinal, lateral and vertical directions are further introduced.

\section{A. Close formation modeling}

It is first envisaged that the Lead UAV is equipped with the Mach, Heading and Altitude hold autopilots, respectively[8].

$$
\begin{aligned}
& \dot{v}_{0}=-\frac{1}{\tau_{v}} v_{0}+\frac{1}{\tau_{v}} v_{0 c} \\
& \ddot{\psi}_{0}=-\left(\frac{1}{\tau_{\psi a}}+\frac{1}{\tau_{\psi b}}\right) \dot{\psi}_{0}-\frac{1}{\tau_{\psi a} \tau_{\psi b}} \psi_{0}+\frac{1}{\tau_{\psi a} \tau_{\psi b}} \psi_{0 c} \\
& \ddot{h}_{0}=-\left(\frac{1}{\tau_{h a}}+\frac{1}{\tau_{h b}}\right) \dot{h}_{0}-\frac{1}{\tau_{h a} \tau_{h b}} h_{0}+\frac{1}{\tau_{h a} \tau_{h b}} h_{0 c}
\end{aligned}
$$

where $v_{0}, \psi_{0}$ and $h_{0}$ are the Lead's velocity, heading angle and altitude respectively, $v_{0 c}, \psi_{0 c}$ and $h_{0 c}$ denote the reference inputs, $\tau_{v}, \tau_{\psi a}, \tau_{\psi b}, \tau_{h a}$ and $\tau_{h b}$ are constant parameters.

According to the property of the Coriolis equation, the velocity of the Lead UAV in the Wing-frame is described as

$$
v_{w l}^{W}=v_{l}^{W}-\omega_{w}^{W} \times R_{w l}^{W}-v_{w}^{W}+\omega_{w}^{W} \times R_{w}^{W}
$$




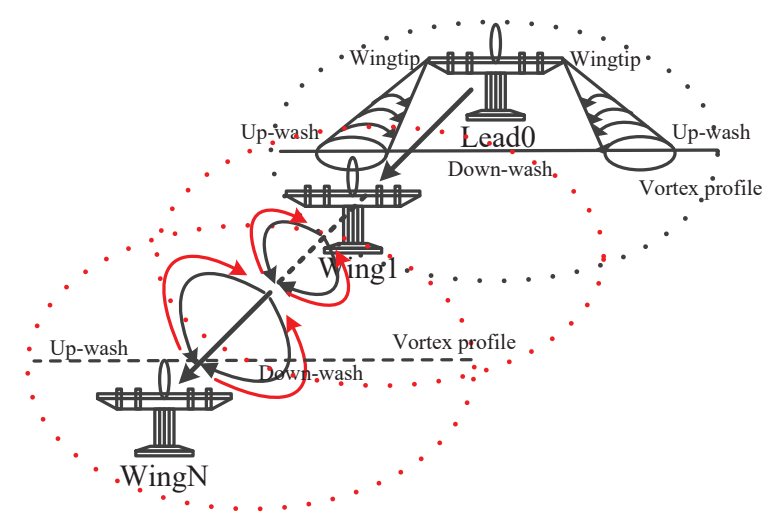

Fig. 1. Close formation geometry with vortex effects and undirected graph.

where $v, \omega$ and $R$ denote the velocity, angular velocity and position, respectively. The superscript $W$ represents the Wing-frame and the subscripts $l$ and $w$ refer to the Lead and Wing UAVs, respectively. The vectors (2) in the longitudinal, lateral and vertical directions are represented as

$$
\omega_{w}^{W}=\left[\begin{array}{c}
0 \\
0 \\
\dot{\psi}_{w}
\end{array}\right], R_{w l}^{W}=\left[\begin{array}{c}
x^{W} \\
y^{W} \\
z^{W}
\end{array}\right], v_{w}^{W}=\left[\begin{array}{c}
v_{w} \\
0 \\
0
\end{array}\right], v_{l}^{L}=\left[\begin{array}{c}
v_{l} \\
0 \\
0
\end{array}\right], R_{w}^{W}=\left[\begin{array}{l}
0 \\
0 \\
0
\end{array}\right]
$$

where $x^{W}, y^{W}$ and $z^{W}$ denote the relative separations between the Lead and Wing UAVs in the Wing-frame. Furthermore, the velocity of the Lead UAV in the Wing-frame is given by $v_{l}^{W}=C^{W L} v_{l}^{L}$ with the rotation matrix $C^{W L}$ from the Lead-frame to the Wing-frame in the following form:

$$
C^{W L}=\left[\begin{array}{ccc}
\cos \left(\psi_{l}-\psi_{w}\right) & -\sin \left(\psi_{l}-\psi_{w}\right) & 0 \\
\sin \left(\psi_{l}-\psi_{w}\right) & \cos \left(\psi_{l}-\psi_{w}\right) & 0 \\
0 & 0 & 1
\end{array}\right]
$$

On substituting (3) and (4) into (2), the nonlinear kinematics in the three directions are given by

$$
\begin{aligned}
& \dot{x}^{W}=v_{l} \cos \left(\psi_{l}-\psi_{w}\right)+\dot{\psi}_{w} y^{W}-v_{w} \\
& \dot{y}^{W}=v_{l} \sin \left(\psi_{l}-\psi_{w}\right)-\dot{\psi}_{w} x^{W} \\
& \dot{z}^{W}=0
\end{aligned}
$$

The flight control of the Wing UAV is essential to be accommodated in close formation geometry to account for aerodynamic coupling vortices from up-washes and side-washes of the Lead UAV. Here, the stability derivatives $\triangle C_{D_{w}}, \triangle C_{L_{w}}$ and $\triangle C_{S_{w}}$ in the Wing's drag, lift and side force are modeled in the following forms[8], [23]:

$$
\begin{aligned}
& \triangle C_{D_{w}}=-\frac{2 C_{L_{l}} C_{L_{w}}}{\pi^{3} A_{R}} \ln \left(\frac{\bar{y}^{2}+\bar{z}^{2}}{(\bar{y}-\pi / 4)^{2}+\bar{z}^{2}} \cdot \frac{\bar{y}^{2}+\bar{z}^{2}}{(\bar{y}+\pi / 4)^{2}+\bar{z}^{2}}\right) \\
& \triangle C_{L_{w}}=\frac{2 a_{w} C_{L_{l}}}{\pi^{3} A_{R}} \ln \left(\frac{\bar{y}^{2}+\bar{z}^{2}}{(\bar{y}-\pi / 4)^{2}+\bar{z}^{2}} \cdot \frac{\bar{y}^{2}+\bar{z}^{2}}{(\bar{y}+\pi / 4)^{2}+\bar{z}^{2}}\right) \\
& \triangle C_{S_{w}}=\frac{\eta S_{v t} a_{v t} C_{L_{l} b}}{2 \pi^{2} A_{R} S h_{z}} \ln \left(\frac{(\bar{y}-\pi / 8)^{2}+\bar{z}^{2}}{(\bar{y}-\pi / 8)^{2}+\left(\bar{z}+h_{z} / b\right)^{2}} \cdot \frac{(\bar{y}+\pi / 8)^{2}+\left(\bar{z}+h_{z} / b\right)^{2}}{(\bar{y}+\pi / 8)^{2}+\bar{z}^{2}}\right)
\end{aligned}
$$

where $\bar{y}=y^{W} / b$ and $\bar{z}=z^{W} / b, C_{L_{l}}$ and $C_{L_{w}}$ denote the lift coefficients of the Lead and Wing UAVs, $a_{w}$ and $a_{v t}$ denote the lift curve slopes of the wing and vertical tail, $A_{R}$ is the aspect ratio of the wing, $\eta$ is the aerodynamic 
efficiency factor of the tail, $S_{v t}$ is the area of the vertical tail, $b$ is the wing span, and $h_{z}$ is the height of the vertical tail.

The existing vortices from the Lead UAV can reduce the induced drag and increase the lift of the Wing UAV in the close formation geometry as shown in Figure 1. To achieve the minimal drag and maximum lift for fuel saving, it is determined that the derivatives of the stabilities are given by

$$
\frac{d \triangle C_{D_{w}}}{d \bar{y}}=\frac{d \triangle C_{D_{w}}}{d \bar{z}}=0, \frac{d \triangle C_{L_{w}}}{d \bar{y}}=\frac{d \triangle C_{L_{w}}}{d \bar{z}}=0
$$

and hence it can be shown that the optimal separations between the Lead and Wing UAVs are $\bar{y}= \pm \pi / 4$ and $\bar{z}=0$ in solving the equality constraints (7). To determine the changes in the drag, lift and side force, linearization is performed on the basis of the optimal close formation geometry with the relative separations in the lateral and vertical directions as $\bar{y}=\pi / 4$ and $\bar{z}=0$.

$$
\begin{aligned}
& p_{D_{w y}}=\frac{\partial \triangle C_{D_{w}}}{\partial \bar{y}} \mid(\bar{y}=\pi / 4, \bar{z}=0)=-\frac{24 C_{L_{l}} C_{L_{w}}}{\pi^{4} A_{R}} \\
& p_{L_{w y}}=\frac{\partial \triangle C_{L_{w}}}{\partial \bar{y}} \mid(\bar{y}=\pi / 4, \bar{z}=0)=\frac{24 a_{w} C_{L_{l}}}{\pi^{4} A_{R}} \\
& p_{S_{w y}}=\frac{\partial \triangle C_{S_{w}}}{\partial \bar{y}} \mid(\bar{y}=\pi / 4, \bar{z}=0)=\frac{\eta S_{v t} a_{v t} h_{z} C_{L_{l}} \mu_{1}}{4 \pi A_{R} S b} \\
& p_{S_{w z}}=\frac{\partial \triangle C_{S_{w}}}{\partial \bar{z}} \mid(\bar{y}=\pi / 4, \bar{z}=0)=-\frac{\eta S_{v t} a_{v t} C_{L_{l}} \mu_{2}}{A_{R} S}
\end{aligned}
$$

where

$$
\mu_{1}=\frac{1}{\left(\pi^{2} / 64\right)\left(\pi^{2} / 64+h_{z}^{2} / b^{2}\right)}-\frac{3}{\left(9 \pi^{2} / 64\right)\left(9 \pi^{2} / 64+h_{z}^{2} / b^{2}\right)}, \mu_{2}=\frac{512 b^{4}}{\left(\pi^{2} b^{2}+64 h_{z}^{2}\right)\left(9 \pi^{2} b^{2}+64 h_{z}^{2}\right)}
$$

and $\partial \triangle C_{D_{w}} / \partial \bar{z}=\partial \triangle C_{L_{w}} / \partial \bar{z} \mid(\bar{y}=\pi / 4, \bar{z}=0)=0$.

On the basis of the formation-hold autopilots of the Lead UAV (1) and the optimal stability derivatives (8), the formation-hold autopilots of the $i$-th Wing UAV in-line $(i=1, \cdots, N)$ as shown in Figure 1 are represented as

$$
\begin{aligned}
& \dot{v}_{i}=-\frac{1}{\tau_{v}} v_{i}+\frac{1}{\tau_{v}} v_{i c}+\frac{q S}{m} \sum_{k=1}^{i} p_{D_{w y}} y_{k, k-1} \\
& \ddot{\psi}_{i}=-\left(\frac{1}{\tau_{\psi a}}+\frac{1}{\tau_{\psi b}}\right) \dot{\psi}_{i}-\frac{1}{\tau_{\psi a} \tau_{\psi b}} \psi_{i}+\frac{1}{\tau_{\psi a} \tau_{\psi b}} \psi_{i c}+\frac{q S}{m} \sum_{k=1}^{i}\left(p_{S_{w y}} y_{k, k-1}+p_{S_{w z}} z_{k, k-1}\right) \\
& \ddot{h}_{i}=-\left(\frac{1}{\tau_{h a}}+\frac{1}{\tau_{h b}}\right) \dot{h}_{i}-\frac{1}{\tau_{h a} \tau_{h b}} h_{i}+\frac{1}{\tau_{h a} \tau_{h b}} h_{i c}+\frac{q S}{m} \sum_{k=1}^{i} p_{L_{w y}} y_{k, k-1}
\end{aligned}
$$

where $y_{i, i-1}$ and $z_{i, i-1}$ denote the relative separations between the $i$-th UAV and the $(i-1)$-th UAV in the lateral and vertical directions, $q$ is the dynamic pressure, $S$ is the surface area of the elliptical wing, and $m$ is the total mass of each UAV.

Define the errors $x_{e i}=x_{c}-x_{i, i-1}, y_{e i}=y_{c}-y_{i, i-1}, z_{e i}=z_{c}-z_{i, i-1}, v_{e i}=v_{i-1}-v_{i}$, and $\psi_{e i}=\psi_{i-1}-\psi_{i}, i=$ $1, \cdots, N$, where $x_{c}, y_{c}$ and $z_{c}$ denote the optimal separations, and $x_{i, i-1}$ denotes the relative separation in the longitudinal direction. Furthermore, define $\xi_{i, i-1}=\dot{z}_{i, i-1}=\dot{h}_{i}-\dot{h}_{i-1}$. On the basis of the optimal separations $x_{c}$ and $y_{c}$, and trimming velocity $v_{c}$, the kinematics of the $i$-th UAV in-line can be rewritten as

$$
\begin{aligned}
& \dot{x}_{i, i-1}=v_{c} \cos \psi_{e i}+\dot{\psi}_{i} y_{c}-v_{i} \\
& \dot{y}_{i, i-1}=v_{c} \sin \psi_{e i}-\dot{\psi}_{i} x_{c} \\
& \dot{\xi}_{i, i-1}=-\left(\frac{1}{\tau_{h a}}+\frac{1}{\tau_{h b}}\right) \xi_{i, i-1}-\frac{1}{\tau_{h a} \tau_{h b}} z_{i, i-1}+\frac{1}{\tau_{h a} \tau_{h b}}\left(h_{i c}-h_{(i-1) c}\right)+\frac{q S}{m} p_{L_{w y}} y_{i, i-1}
\end{aligned}
$$

Remark 2.1: (i) The vortex effects are difficult to measure and model. The strongly nonlinear and coupling characteristics in CFF can be represented by nonlinear but linearly parameterized functions [5], [8] or be treated as unknown functions in a nonparametric form [9]. Real-time accurate knowledge of the aerodynamic effects in CFF 
is generally unavailable; thus, linearization based on the optimal CFF geometry is used in this study. (ii) Unlike the directed networks communicating with one preceding UAV through sensors[9], black and red arrows in Figure 1 show the undirected data transmission amongst the neighboring Wing UAVs (e.g., interval position, velocity, heading angle and angular velocity information).

\section{B. Simultaneous actuator and sensor fault modeling}

Define the input vectors $u_{i X}, u_{i Y}$ and $u_{i Z}$, state vectors $x_{i X}, x_{i Y}$ and $x_{i Z}$, output vectors $y_{i X}, y_{i Y}$ and $y_{i Z}$, system uncertainties $d_{i X}, d_{i Y}$ and $d_{i Z}$, and nonlinear item $g\left(x_{i Y}\right)$ for the Mach, Heading and Altitude hold autopilots, i.e., $(X, Y, Z)$ channels in the longitudinal, lateral and vertical directions.

$$
\begin{aligned}
& u_{i X}=v_{i c}, u_{i Y}=\psi_{i c}, u_{i Z}=h_{i c} \\
& x_{i X}=\left[\begin{array}{llll}
x_{i, i-1} & x_{e i} / s & v_{i} & v_{e i} / s
\end{array}\right]^{T}, x_{i Y}=\left[\begin{array}{llll}
y_{i, i-1} & y_{e i} / s & \psi_{i} & \psi_{e i} / s \\
\psi_{i}
\end{array}\right]^{T}, x_{i Z}=\left[\begin{array}{lll}
z_{i, i-1} & \xi_{i, i-1} & z_{e i} / s
\end{array}\right]^{T} \\
& d_{i X}=\left[\begin{array}{llll}
x_{c} & v_{i-1} & \sum_{k=1}^{i} y_{k, k-1} & \dot{\psi}_{i} \cos \psi_{e i}
\end{array}\right]^{T}, d_{i Y}=\left[\begin{array}{llll}
y_{c} & \psi_{i-1} & \sum_{k=1}^{i} z_{k, k-1} & \sum_{k=1}^{i} y_{k, k-1}
\end{array}\right]^{T} \\
& d_{i Z}=\left[h_{(i-1) c} z_{c} y_{i, i-1}\right]^{T} \\
& y_{i X}=\left[\begin{array}{ll}
x_{i, i-1} & v_{i}
\end{array}\right]^{T}, y_{i Y}=\left[y_{i, i-1} \psi_{i} \dot{\psi}_{i}\right]^{T}, y_{i Z}=z_{i, i-1}, g\left(x_{i Y}\right)=\left[\begin{array}{lllll}
v_{c} \sin \psi_{e i} & 0 & 0 & 0 & 0
\end{array}\right]^{T}
\end{aligned}
$$

Assume that each UAV suffers from additive actuator and sensor faults, the dynamic models of the $i$-th UAV in-line $(i=1, \cdots, N)$ in the $X, Y$ and $Z$ channels are described as

$$
\begin{gathered}
\dot{x}_{i X}=A_{X} x_{i X}+B_{X}\left(u_{i X}+f_{v i}^{a}\right)+D_{X} d_{i X} \\
y_{i X}=C_{X} x_{i X}+F_{s X} f_{i X}^{s} \\
\dot{x}_{i Y}=A_{Y} x_{i Y}+B_{Y}\left(u_{i Y}+f_{\psi i}^{a}\right)+D_{Y} d_{i Y}+g\left(x_{i Y}\right) \\
y_{i Y}=C_{Y} x_{i Y}+F_{s Y} f_{i Y}^{s} \\
\dot{x}_{i Z}=A_{Z} x_{i Z}+B_{Z}\left(u_{i Z}+f_{h i}^{a}+f_{h(i-1)}^{a}\right)+D_{Z} d_{i Z} \\
y_{i Z}=C_{Z} x_{i Z}+F_{s Z} f_{i Z}^{s}
\end{gathered}
$$

where scalars $f_{v i}^{a}, f_{\psi i}^{a}$ and $f_{h i}^{a}$ denote the additive actuator faults of the $i$-th $\mathrm{UAV}$ in the $X, Y$ and $Z$ input channels, $f_{h(i-1)}^{a}$ denotes the additive actuator fault of the $(i-1)$-th UAV in the $Z$ input channel, $f_{i X}^{s} \in R^{q_{X i}}, f_{i Y}^{s} \in R^{q_{Y i}}$ and $f_{i Z}^{s} \in R^{q_{Z i}}$ denote the sensor faults in the output channels. Matrices $A_{X}, B_{X}, D_{X}, A_{Y}, B_{Y}, D_{Y}, A_{Z}, B_{Z}$ and $D_{Z}$ are appropriate gains under specific flight conditions in (9) and (10). Matrices $C_{X}, F_{s X}, C_{Y}, F_{s Y}, C_{Z}$ and $F_{s Z}$ are of known and appropriate dimensions.

Remark 2.2: (i) It is verified that the close formation systems (12)-(14) are controllable and observable. (ii) The physical meaning of actuator fault is the deviation of the reference control input. These actuator faults $f_{v i}^{a}, f_{\psi i}^{a}$ and $f_{h i}^{a}$ may cause additive disturbances in the low-level autopilot's response to velocity, heading angle and altitude commands $v_{i c}, \psi_{i c}$ and $h_{i c}$ in CFF. The physical meaning of sensor fault refers to the deviation of the sensor output, such as the position errors $x_{i, i-1}, y_{i, i-1}$ and $z_{i, i-1}$ by GPS measuring, the heading angle $\psi_{i}$ and heading angular acceleration $\dot{\psi}_{i}$ errors. The control surface actuator faults and the sensor errors caused by hardware or cyber attacks 
are not considered herein. (iii) The nonlinear term $g\left(x_{i Y}\right)$ in the $Y$ channel of the $i$-th UAV in-line satisfies the Lipschitz constraint, i.e., $\left\|g\left(x_{i Y}\right)-g(x)\right\| \leq L_{g}\left\|x_{i Y}-x\right\|$, where $L_{g}=v_{c}$ is the Lipschitz constant. Moreover, the initial condition $g(0)=0$ is assumed for simplicity.

Definition 2.1 [33]: Let $\gamma>0$ and $\epsilon>0$ be given constants, the closed-loop system can achieve a $H_{\infty}$ performance index no larger than $\gamma$, i.e., $\left\|G_{z d}\right\|<\gamma$ if the following form holds:

$$
\int_{0}^{\infty} z^{T}(t) z(t) d t \leq \gamma^{2} \int_{0}^{\infty} d(t)^{T} d(t) d t+\epsilon
$$

Lemma 2.1[34]: There exists a zero eigenvalue for the Laplacian matrix $\mathcal{L}$ with $1_{N}$ as a corresponding right eigenvector and all nonzero eigenvalues have positive real parts in the undirected graph $\mathcal{G}$. Assume that $\lambda_{i}$ denotes the $i$-th eigenvalue of $\mathcal{L}$, thus, $0=\lambda_{1}<\lambda_{2} \leq \cdots \leq \lambda_{N}$. Furthermore, if $1_{N}^{T} X=0$, then $X^{T} \mathcal{L} X \geq \lambda_{2} X^{T} X$.

Control objective: This study aims to stabilize the dynamics of the $i$-th UAV in-line (12)-(14) in the $X, Y$ and $Z$ channels through an FE/FTC design involving (i) the decentralized FE protocol to estimate the state and fault information, and (ii) the distributed FTC protocol based on estimated information and relative output information of neighbors. Furthermore, the proposed controllers in CFF models are developed so that the Wing UAV's velocity, heading angle to track with the relative signals of the Lead UAV and separations in the longitudinal, lateral and vertical directions are invariable while the Lead UAV is being maneuvered.

\section{Decentralized fault estimation Design}

Define the extended states and system uncertainties as

$$
\begin{gathered}
\bar{x}_{i X}=\left[\begin{array}{c}
x_{i X} \\
f_{v i}^{a} \\
f_{i X}^{s}
\end{array}\right], \bar{x}_{i Y}=\left[\begin{array}{c}
x_{i Y} \\
f_{\psi i}^{a} \\
f_{i Y}^{s}
\end{array}\right], \bar{x}_{i Z}=\left[\begin{array}{c}
x_{i Z} \\
f_{h i}^{a} \\
f_{h(i-1)}^{a} \\
f_{i Z}^{s}
\end{array}\right] \\
\bar{d}_{i X}=\left[\begin{array}{c}
d_{i X} \\
\dot{f}_{v i}^{a} \\
\dot{f}_{i X}^{s}
\end{array}\right], \bar{d}_{i Y}=\left[\begin{array}{c}
d_{i Y} \\
\dot{f}_{\psi i}^{a} \\
\dot{f}_{i Y}^{s}
\end{array}\right], \bar{d}_{i Z}=\left[\begin{array}{c}
d_{i Z} \\
\dot{f}_{h i}^{a} \\
\dot{f}_{h(i-1)}^{a} \\
\dot{f}_{i Z}^{s}
\end{array}\right]
\end{gathered}
$$

and augment the dynamics of the $i$-th UAV (12)-(14) into

$$
\begin{gathered}
\dot{\bar{x}}_{i j}=\bar{A}_{j} \bar{x}_{i j}+\bar{B}_{j} u_{i j}+\bar{D}_{j} \bar{d}_{i j} \\
y_{i j}=\bar{C}_{j} \bar{x}_{i j}, j=X, Z \\
\dot{\bar{x}}_{i Y}=\bar{A}_{Y} \bar{x}_{i Y}+\bar{B}_{Y} u_{i Y}+\bar{D}_{Y} \bar{d}_{i Y}+\bar{g}\left(A_{0} \bar{x}_{i Y}\right) \\
y_{i Y}=\bar{C}_{Y} \bar{x}_{i Y}
\end{gathered}
$$


where the gain matrices are described as

$$
\begin{aligned}
& \bar{A}_{X}=\left[\begin{array}{ccc}
A_{X} & B_{X} & 0_{4 \times q_{X}} \\
0_{\left(1+q_{X i}\right) \times 4} & 0_{\left(1+q_{X i}\right) \times 1} & 0_{\left(1+q_{X i}\right) \times q_{X i}}
\end{array}\right], \bar{A}_{Y}=\left[\begin{array}{ccc}
A_{Y} & B_{Y} & 0_{5 \times q_{Y i}} \\
0_{\left(1+q_{Y i}\right) \times 5} & 0_{\left(1+q_{Y i}\right) \times 1} & 0_{\left(1+q_{Y i}\right) \times q_{Y i}}
\end{array}\right] \\
& \bar{A}_{Z}=\left[\begin{array}{cccc}
A_{Z} & B_{Z} & B_{Z} & 0_{3 \times q_{Z i}} \\
0_{\left(2+q_{Z i}\right) \times 3} & 0_{\left(2+q_{Z i}\right) \times 1} & 0_{\left(2+q_{Z i}\right) \times 1} & 0_{\left(2+q_{Z i}\right) \times q_{Z i}}
\end{array}\right] \\
& \bar{B}_{X}=\left[\begin{array}{c}
B_{X} \\
0_{\left(1+q_{X i}\right) \times 1}
\end{array}\right], \bar{B}_{Y}=\left[\begin{array}{c}
B_{Y} \\
0_{\left(1+q_{Y i}\right) \times 1}
\end{array}\right], \bar{B}_{Z}=\left[\begin{array}{c}
B_{Z} \\
0_{\left(2+q_{Z i}\right) \times 1}
\end{array}\right], \bar{g}\left(A_{0} \bar{x}_{i Y}\right)=\left[\begin{array}{c}
g\left(x_{i Y}\right) \\
0_{\left(1+q_{Y i}\right) \times 1}
\end{array}\right] \\
& \bar{D}_{X}=\operatorname{diag}\left(D_{Z}, 1, I_{q_{X i}}\right), \bar{C}_{X}=\left[\begin{array}{lll}
C_{X} & 0 & F_{s X}
\end{array}\right] \\
& \bar{D}_{Y}=\operatorname{diag}\left(D_{Y}, 1, I_{q_{Y i}}\right), \bar{C}_{Y}=\left[\begin{array}{lll}
C_{Y} & 0 & F_{s Y}
\end{array}\right] \\
& \bar{D}_{Z}=\operatorname{diag}\left(D_{Z}, 1,1, I_{q_{Z i}}\right), \bar{C}_{Z}=\left[\begin{array}{llll}
C_{Z} & 0 & 0 & F_{s Z}
\end{array}\right]
\end{aligned}
$$

and $A_{0}=\left[\begin{array}{ll}I_{5} & 0_{5 \times\left(1+q_{Y i}\right)}\end{array}\right]$. Furthermore, the subscript $j$ represents the $X$ and $Z$ channels, respectively.

The augmented dynamics $(17)$ is the special case of the dynamics $(18)$ when $\bar{g}\left(A_{0} \bar{x}_{i Y}\right)=\left[\begin{array}{llll}0_{1 \times 5} & 0_{1 \times 1} & 0_{1 \times q_{Y}}\end{array}\right]^{T}$ is satisfied. Furthermore, given the accessible output information rather than the state information in the real-time applications, the dynamics (18) is taken into consideration. Thus, the state $\bar{x}_{i Y}$ of the $i$-th dynamic UAV model in the $Y$ channel needs to be estimated by the $i$-th unknown input observer in the decentralized fashion, which means that the designed observer only requires the information from the corresponding UAV rather than its neighboring observers.

$$
\begin{aligned}
& \dot{z}_{i Y}=M_{Y} z_{i Y}+G_{Y} u_{i Y}+J_{Y} y_{i Y}+\Gamma_{Y} \bar{g}\left(A_{0} \hat{\bar{x}}_{i Y}\right) \\
& \hat{\bar{x}}_{i Y}=z_{i Y}+H_{Y} y_{i Y}
\end{aligned}
$$

where $z_{i Y} \in R^{6+q_{Y i}}$ is the state of the $i$-th unknown input observer, $\hat{\bar{x}}_{i Y}=\left[\hat{x}_{i Y}^{T}, \hat{f}_{\psi i}^{a T}, \hat{f}_{i Y}^{s T}\right]^{T}$ is the estimate of the extended state $\bar{x}_{i Y}$ in the $Y$ channel, $\hat{x}_{i Y}, \hat{f}_{\psi i}^{a}$ and $\hat{f}_{i Y}^{s}$ are the estimates of the respective state $x_{i Y}$, the actuator fault $f_{\psi i}^{a}$, and the sensor fault $f_{i Y}^{s}$. Matrices $M_{Y}, G_{Y}, J_{Y}$ and $H_{Y}$ are of appropriate dimensions to be derived. Furthermore, define the estimation error as $e_{i Y}=\bar{x}_{i Y}-\hat{\bar{x}}_{i Y}=\left[\begin{array}{lll}e_{i Y}^{x T} & e_{i Y}^{a T} & e_{i Y}^{s T}\end{array}\right]^{T}$ with $e_{i Y}^{x}=x_{i Y}-\hat{x}_{i Y}, e_{i Y}^{a}=$ $f_{\psi i}^{a}-\hat{f}_{\psi i}^{a}$, and $e_{i Y}^{s}=f_{i Y}^{s}-\hat{f}_{i Y}^{s}$. Then, matrices $\Gamma_{Y}$ and $J_{Y}$ are defined as $\Gamma_{Y}=I_{6+q_{Y i}}-H_{Y} \bar{C}_{Y}$ and $J_{Y}=J_{1 Y}+J_{2 Y}$ in order to decouple the effects of states, system uncertainties and nonlinear items.

$$
\begin{aligned}
\dot{e}_{i Y}= & \left(\Gamma_{Y} \bar{A}_{Y}-J_{1 Y} \bar{C}_{Y}\right) e_{i Y}+\left(\Gamma_{Y} \bar{B}_{Y}-G_{Y}\right) u_{i Y}+\left(\left(\Gamma_{Y} \bar{A}_{Y}-J_{1 Y} \bar{C}_{Y}\right) H_{Y}-J_{2 Y}\right) y_{i Y} \\
& +\Gamma_{Y} \bar{D}_{Y} \bar{d}_{i Y}+\left(\Gamma_{Y} \bar{A}_{Y}-J_{1 Y} \bar{C}_{Y}-M_{Y}\right) z_{i Y}+\Gamma_{Y} \bar{g}\left(A_{0} \bar{x}_{i Y}\right)-\Gamma_{Y} \bar{g}\left(A_{0} \hat{\bar{x}}_{i Y}\right)
\end{aligned}
$$

Then, with the following equation constraints

$$
\begin{gathered}
M_{Y} \text { is Hurwitz } \\
\Gamma_{Y} \bar{A}_{Y}-J_{1 Y} \bar{C}_{Y}=M_{Y} \\
\left(\Gamma_{Y} \bar{A}_{Y}-J_{1 Y} \bar{C}_{Y}\right) H_{Y}=J_{2 Y} \\
\Gamma_{Y} \bar{B}_{Y}=G_{Y}
\end{gathered}
$$

the $i$-th estimation error dynamics are obtained as

$$
\dot{e}_{i Y}=M_{Y} e_{i Y}+\Gamma_{Y} \bar{D}_{Y} \bar{d}_{i Y}+\Gamma_{Y} \Delta \bar{g}_{i}
$$


where $\Delta \bar{g}_{i}=\bar{g}\left(A_{0} \bar{x}_{i Y}\right)-\bar{g}\left(A_{0} \hat{\bar{x}}_{i Y}\right)$.

The designed matrices $M_{Y}, G_{Y}$ and $J_{2 Y}$ can be obtained with the derived matrices $J_{1 Y}$ and $H_{Y}$. Furthermore, define $e_{Y}=\left[e_{1 Y}^{T}, \cdots, e_{N Y}^{T}\right]^{T}, \bar{d}_{Y}=\left[\bar{d}_{1 Y}^{T}, \cdots, \bar{d}_{N Y}^{T}\right]^{T}$, and $\Delta \bar{g}=\left[\Delta \bar{g}_{1}^{T}, \cdots, \Delta \bar{g}_{N}^{T}\right]^{T}$ with $\bar{x}_{Y}=\left[\bar{x}_{1 Y}^{T}, \cdots, \bar{x}_{N Y}^{T}\right]^{T}$ and $\hat{\bar{x}}_{Y}=\left[\hat{\bar{x}}_{1 Y}^{T}, \cdots, \hat{\bar{x}}_{N Y}^{T}\right]^{T}$, and it follows that

$$
\dot{e}_{Y}=\left(I_{N} \otimes\left(\Gamma_{Y} \bar{A}_{Y}-J_{1 Y} \bar{C}_{Y}\right)\right) e_{Y}+\left(I_{N} \otimes \Gamma_{Y} \bar{D}_{Y}\right) \bar{d}_{Y}+\left(I_{N} \otimes \Gamma_{Y}\right) \Delta \bar{g}
$$

Here, a sufficient condition for the existence of a robust unknown input observer (19) is given.

Theorem 3.1: There exists a robust unknown input observer (19) if the estimation error system (25) is robustly asymptotically stable with the constraints in (21)-(24).

Proof: With the definitions in (21)-(24), the estimation error system (25) is equivalent to the original estimation error dynamics (20). Hence, if (25) is robustly asymptotically stable, then (20) is also robustly asymptotically stable, indicating that $\lim _{t \rightarrow \infty} e_{i Y}=0$ in the presence of system uncertainties and nonlinear items. Furthermore, the objective of obtaining the unknown input observer is to design $H_{Y}$ and $J_{1 Y}$ such that (26) is robustly asymptotically stable.

Remark 3.1: (i) The $i$-th estimation error dynamics can be completely decoupled when the terms $\Gamma_{Y} \bar{D}_{Y} \bar{d}_{i Y}=0$ and $\Gamma_{Y} \Delta \bar{g}_{i}=0$ are satisfied. The Hurwitz condition of the matrix $M_{Y}$ ensures that (26) is robustly asymptotically stable. However, (25) and (26) show that the FE performance is influenced by the system uncertainty $\bar{d}_{i Y}$ and the nonlinear error $\Delta \bar{g}_{i}$. (ii) The prior information of the nonlinear error $\Delta \bar{g}_{i}$ and actuator and sensor faults in the system uncertainty $\bar{d}_{i Y}$ is not required in this study. This positive effect is evident compared with the assumptions of bounded system uncertainties and nonlinearities[17], [24], [32]. (iii) Unlike the Luenberger observer, which generates residual signals and fault estimators to detect, isolate and estimate the faults[20], unknown input observers are proposed in this study. The system uncertainty $\bar{d}_{i Y}$ and the nonlinear error $\Delta \bar{g}_{i}$ can be dealt with instead of being decoupled by the following separated and integrated FE/FTC strategies.

\section{Distributed Fault-TOlerant CONTROL Design}

In this section, the undirected topology $\mathcal{G}$ in Figure 1 implies that each Wing UAV in-line can receive the relative output information rather than the state information of its neighboring Wing UAVs. On the basis of the estimated information in the unknown input observers (19) and the relative output information of neighbors, two distributed protocols are put forward, namely, the separated FE/FTC and the integrated FE/FTC designs.

Consider that the $Y$ channel represents the general description, the distributed fault-tolerant controller for the $i$-th UAV in the lateral direction is designed as

$$
u_{i Y}=-K_{Y} \hat{\bar{x}}_{i Y}-g_{Y} K_{g Y} \sum_{j=1}^{N} a_{i j}\left(y_{i Y}-F_{s Y} \hat{f}_{i Y}^{s}-y_{j Y}+F_{s Y} \hat{f}_{j Y}^{s}\right)
$$

where $K_{Y}=\left[\begin{array}{lll}K_{x Y} & 1 & 0_{1 \times q_{Y i}}\end{array}\right]$ denotes the augmented gain with the state feedback gain $K_{x Y} \in R^{1 \times 5}, a_{i j}$ denotes the $(i, j)$-th entry of the adjacency matrix $\mathcal{A}, K_{g Y} \in R^{1 \times 3}$ denotes the distributed gain and $g_{Y}$ is a positive scalar.

Then, the closed-loop system (13) is rewritten as

$$
\begin{aligned}
\dot{x}_{i Y} & =\left(A_{Y}-B_{Y} K_{x Y}\right) x_{i Y}+B_{Y} K_{Y} e_{i Y}+g\left(x_{i Y}\right)+D_{Y} d_{i Y} \\
& -g_{Y} B_{Y} K_{g Y} \sum_{j=1}^{N} a_{i j}\left(C_{Y}\left(x_{i Y}-x_{j Y}\right)+F_{s Y}\left(e_{i Y}^{s}-e_{j Y}^{s}\right)\right)
\end{aligned}
$$




\section{A. Separated FE and FTC design}

Note that the estimation errors in the decentralized FE system are not considered in the following separated FTC system, thus the corresponding FTC system (28) with the distributed controller (27) is derived as

$$
\left\{\begin{array}{l}
\dot{x}_{Y}=\left(I_{N} \otimes\left(A_{Y}-B_{Y} K_{x Y}\right)-g_{Y} \mathcal{L} \otimes B_{Y} K_{g Y} C_{Y}\right) x_{Y}+\left(I_{N} \otimes D_{Y}\right) d_{Y}+g\left(x_{Y}\right) \\
z_{Y 1}=C_{x Y} x_{Y}
\end{array}\right.
$$

where $d_{Y}=\left[d_{1 Y}^{T}, \cdots, d_{N Y}^{T}\right]^{T}, x_{Y}=\left[x_{1 Y}^{T}, \cdots, x_{N Y}^{T}\right]^{T}$ and $g\left(x_{Y}\right)=\left[g\left(x_{1 Y}\right)^{T}, \cdots, g\left(x_{N Y}\right)^{T}\right]^{T}, z_{Y 1} \in R^{r_{Y 1} N}$ is the accessible output in order to verify the separated FTC performance with the matrix $C_{x Y} \in R^{r_{Y 1} N \times 5 N}, \mathcal{L}$ is the Laplacian matrix corresponding to the undirected graph $\mathcal{G}$. Hence, the objective of the separated FTC design is to devise the state feedback gain $K_{x Y}$ and the distributed gain $K_{g Y}$ to guarantee the robust stability of the separated FTC system (29).

Theorem 4.1: Given positive scalars $\gamma_{1}$ and $\varepsilon_{Y 1}$, matrix $C_{x Y 0} \in R^{r_{Y 1} \times 5}$, the separated FTC system (29) with the distributed controller $(27)$ is stable with the $H_{\infty}$ performance $\left\|G_{z_{Y 1} d_{Y}}\right\|<\gamma_{1}$, if there exists a symmetric positive-definite matrix $Q_{Y 0} \in R^{5 \times 5}$, and matrices $X_{1} \in R^{1 \times 5}$ and $X_{2} \in R^{1 \times 3}$ such that

$$
\left[\begin{array}{cccc}
\Omega_{1} & I_{N} \otimes Q_{Y 0} D_{Y} & I_{N} \otimes Q_{Y 0} & I_{N} \otimes C_{x Y 0}^{T} \\
\star & -\gamma_{1}^{2} I_{4 N} & 0 & 0 \\
\star & \star & -\varepsilon_{Y 1} I_{5 N} & 0 \\
\star & \star & \star & -I_{r_{Y 1} N}
\end{array}\right]<0
$$

with $\Omega_{1}=I_{N} \otimes \operatorname{He}\left(Q_{Y 0} A_{Y}-B_{Y} X_{1}\right)+\varepsilon_{Y 1} L_{g}^{2} I_{5 N}-\mathcal{L} \otimes \operatorname{He}\left(g_{Y} B_{Y} X_{2} C_{Y}\right)$. Then, the state feedback gain is given by $K_{x Y}=\hat{Q}_{Y 0}^{-1} X_{1}$, and the distributed gain is given by $K_{g Y}=\hat{Q}_{Y 0}^{-1} X_{2}$ with $Q_{Y 0} B_{Y}=B_{Y} \hat{Q}_{Y 0}$.

Proof: Consider a Lyapunov function $V_{x_{Y}}=x_{Y}^{T} Q_{Y} x_{Y}$ with a symmetric positive-definite matrix $Q_{Y}$, and the time derivative of $V_{x_{Y}}$ is obtained with a positive scalar $\varepsilon_{Y 1}$.

$$
\begin{aligned}
\dot{V}_{x_{Y}} & \leq x_{Y}^{T} \operatorname{He}\left(Q_{Y}\left(I_{N} \otimes\left(A_{Y}-B_{Y} K_{x Y}\right)-g_{Y} \mathcal{L} \otimes B_{Y} K_{g Y} C_{Y}\right)\right) x_{Y}+\operatorname{He}\left(x_{Y}^{T} Q_{Y}\left(I_{N} \otimes D_{Y}\right) d_{Y}\right) \\
& +\varepsilon_{Y 1}^{-1} x_{Y}^{T} Q_{Y} Q_{Y}^{T} x_{Y}+\varepsilon_{Y 1} g^{T}\left(x_{Y}\right) g\left(x_{Y}\right)
\end{aligned}
$$

On the basis of the condition $g(0)=0$, then $\left\|g\left(x_{Y}\right)\right\| \leq L_{g}\left\|x_{Y}\right\|$. According to Definition 2.1, the sufficient condition of achieving the $H_{\infty}$ performance $\left\|G_{z_{Y 1} d_{Y}}\right\|<\gamma_{1}$ is $z_{Y 1}^{T} z_{Y 1}-\gamma_{1}^{2} d_{Y}^{T} d_{Y}+\dot{V}_{x Y}<0$. Denote $C_{x Y}=$ $I_{N} \otimes C_{x Y 0}$ and $Q_{Y}=I_{N} \otimes Q_{Y 0}$ with the symmetric positive-definite matrix $Q_{Y 0}$. According to the condition $Q_{Y 0} B_{Y}=B_{Y} \hat{Q}_{Y 0}, K_{x Y}=\hat{Q}_{Y 0}^{-1} X_{1}$ and $K_{g Y}=\hat{Q}_{Y 0}^{-1} X_{2}$, the Schur Lemma is used to obtain the LMI (30). This completes the proof.

Furthermore, it is shown that the nonlinear error $\Delta \bar{g}$ in the FTC system is not considered in the following separated FE system, thus the corresponding FE system (26) becomes

$$
\left\{\begin{array}{l}
\dot{e}_{Y}=\left(I_{N} \otimes\left(\Gamma_{Y} \bar{A}_{Y}-J_{1 Y} \bar{C}_{Y}\right)\right) e_{Y}+\left(I_{N} \otimes \Gamma_{Y} \bar{D}_{Y}\right) \bar{d}_{Y} \\
z_{Y 2}=C_{e Y} e_{Y}
\end{array}\right.
$$

where $z_{Y 2} \in R^{r_{Y 2} N}$ is the measured output with $C_{e Y} \in R^{r_{Y 2} N \times\left(6+q_{Y i}\right) N}$. Hence, the objective of the proposed FE design is to devise the gains $H_{Y}$ and $J_{1 Y}$ to guarantee the robust stability of the separated FE system (32). 
Theorem 4.2: Given a positive scalar $\gamma_{2}$, matrices $C_{e Y x} \in R^{r_{Y 2} \times 5}, C_{e Y a} \in R^{r_{Y 2} \times 1}$ and $C_{e Y s} \in R^{r_{Y 2} \times q_{Y i}}$, the separated FE system (32) is stable with the $H_{\infty}$ performance $\left\|G_{z_{Y 2} \bar{d}_{Y}}\right\|<\gamma_{2}$, if there exist symmetric positivedefinite matrices $P_{Y 1} \in R^{5 \times 5}, P_{Y 2} \in R^{1 \times 1}$ and $P_{Y 3} \in R^{q_{Y i} \times q_{Y i}}$, and matrices $X_{3} \in R^{5 \times 3}, X_{4} \in R^{5 \times 3}, X_{5} \in$ $R^{1 \times 3}, X_{6} \in R^{1 \times 3}, X_{7} \in R^{q_{Y i} \times 3}$ and $X_{8} \in R^{q_{Y i} \times 3}$ such that

$$
\left[\begin{array}{ccc}
\Omega_{2} & \Omega_{3} & I_{N} \otimes\left[C_{e Y x} C_{e Y a} C_{e Y s}\right]^{T} \\
\star & -\gamma_{2}^{2} I_{\left(5+q_{Y i}\right) N} & 0 \\
\star & \star & -I_{r_{Y 2} N}
\end{array}\right]<0
$$

with

$$
\begin{gathered}
\Omega_{2}=I_{N} \otimes\left[\begin{array}{ccc}
\Omega_{211} & \Omega_{212} & \Omega_{213} \\
\star & \operatorname{He}\left(-X_{5} C_{Y} B_{Y}\right) & \Omega_{223} \\
\star & \star & \operatorname{He}\left(-X_{8} F_{s Y}\right)
\end{array}\right], \Omega_{3}=I_{N} \otimes\left[\begin{array}{ccc}
\Omega_{311} & 0 & -X_{3} F_{s Y} \\
-X_{5} C_{Y} D_{Y} & P_{Y 2} & -X_{5} F_{s Y} \\
-X_{7} C_{Y} D_{Y} & 0 & P_{Y 3}-X_{7} F_{s Y}
\end{array}\right] \\
\Omega_{211}=\operatorname{He}\left(P_{Y 1} A_{Y}-X_{3} C_{Y} A_{Y}-X_{4} C_{Y}\right), \Omega_{212}=P_{Y 1} B_{Y}-X_{3} C_{Y} B_{Y}-A_{Y}^{T} C_{Y}^{T} X_{5}^{T}-C_{Y}^{T} X_{6}^{T} \\
\Omega_{213}=-X_{4} F_{s Y}-A_{Y}^{T} C_{Y}^{T} X_{7}^{T}-C_{Y}^{T} X_{8}^{T}, \Omega_{223}=-X_{6} F_{s Y}-B_{Y}^{T} C_{Y}^{T} X_{7}^{T}, \Omega_{311}=P_{Y 1} D_{Y}-X_{3} C_{Y} D_{Y}
\end{gathered}
$$

Then, the unknown input observer gains are given by $H_{Y 1}=P_{Y 1}^{-1} X_{3}, J_{1 Y 1}=P_{Y 1}^{-1} X_{4}, H_{Y 2}=P_{Y 2}^{-1} X_{5}, J_{1 Y 2}=$ $P_{Y 2}^{-1} X_{6}, H_{Y 3}=P_{Y 3}^{-1} X_{7}$, and $J_{1 Y 3}=P_{Y 3}^{-1} X_{8}$.

Proof: Consider a Lyapunov function $V_{e_{Y}}=e_{Y}^{T} P_{Y} e_{Y}$ with a symmetric positive-definite matrix $P_{Y}$, and the time derivative of $V_{e_{Y}}$ is obtained as

$$
\dot{V}_{e Y}=e_{Y}^{T} \operatorname{He}\left(P_{Y}\left(I_{N} \otimes\left(\Gamma_{Y} \bar{A}_{Y}-J_{1 Y} \bar{C}_{Y}\right)\right)\right) e_{Y}+\operatorname{He}\left(e_{Y}^{T} P_{Y}\left(I_{N} \otimes \Gamma_{Y} \bar{D}_{Y}\right) \bar{d}_{Y}\right)
$$

Denote $C_{e Y}=I_{N} \otimes\left[\begin{array}{lll}C_{e Y x} & C_{e Y a} & C_{e Y s}\end{array}\right], P_{Y}=I_{N} \otimes \operatorname{diag}\left\{P_{Y 1} P_{Y 2} P_{Y 3}\right\}$ with symmetric positive-definite matrices $P_{Y 1}, P_{Y 2}$ and $P_{Y 3}$. Define $H_{Y}=\left[\begin{array}{lll}H_{Y 1}^{T} & H_{Y 2}^{T} & H_{Y 3}^{T}\end{array}\right]^{T}$ and $J_{1 Y}=\left[\begin{array}{lll}J_{1 Y 1}^{T} & J_{1 Y 2}^{T} & J_{1 Y 3}^{T}\end{array}\right]^{T}$. According to Definition 2.1, the sufficient condition of achieving the $H_{\infty}$ performance $\left\|G_{z_{Y 2} \bar{d}_{Y}}\right\|<\gamma_{2}$ is $z_{Y 2}^{T} z_{Y 2}-\gamma_{2}^{2} \bar{d}_{Y}^{T} \bar{d}_{Y}+$ $\dot{V}_{e Y}<0$. Thus, the Schur Lemma is applied and the proof of Theorem 4.2 is straightforward and is omitted here.

Remark 4.1: (i) Graph theory is adopted to describe undirected transformation networks from an arbitrary connected topology[10] to the CFF networks in this study. (ii) Unlike the integration of fault detection and FTC mechanisms, which uses residuals between sensor measurements and desired values from monitors for detecting fault occurrence[14], [25], the proposed FE/FTC control scheme does not utilize any fault detection and isolation information to detect, identify and isolate faults. As a result, online computation is minimized and the responsiveness of distributed controllers is expedited. (iii) Unlike the design based on local state information[29], FE information[17], [24], [31] or only output estimation errors[19], [21], the proposed FTC scheme (27) is constructed in a fully distributed fashion based on estimated information in FE and on the output information of neighbors.

\section{B. Integrated FE and FTC design}

Note that the bidirectional interactions exist in both the FE and FTC systems. The estimation error $e_{Y}$ from the FE process influences the FTC performance and the nonlinear error $\Delta \bar{g}$ from the FTC process influences the FE 
performance in turn. It follows that the integrated FE/FTC model is derived as

$$
\left\{\begin{aligned}
\dot{x}_{Y}= & \left(I_{N} \otimes\left(A_{Y}-B_{Y} K_{x Y}\right)-g_{Y} \mathcal{L} \otimes B_{Y} K_{g Y} C_{Y}\right) x_{Y} \\
& +\left(I_{N} \times B_{Y} K_{Y}-g_{Y} \mathcal{L} \otimes B_{Y} K_{g Y} F_{s Y} S_{1}\right) e_{Y}+\left(I_{N} \otimes D_{Y} S_{2}\right) \bar{d}_{Y}+g\left(x_{Y}\right) \\
\dot{e}_{Y}= & \left(I_{N} \otimes\left(\Gamma_{Y} \bar{A}_{Y}-J_{1 Y} \bar{C}_{Y}\right)\right) e_{Y}+\left(I_{N} \otimes \Gamma_{Y} \bar{D}_{Y}\right) \bar{d}_{Y}+\left(I_{N} \otimes \Gamma_{Y}\right) \Delta \bar{g} \\
z_{Y}= & \bar{C}_{x Y} x_{Y}+\bar{C}_{e Y} e_{Y}
\end{aligned}\right.
$$

where $S_{1}=\left[\begin{array}{llll}0_{q_{Y i} \times 5} & 0_{q_{Y i} \times 1} & I_{q_{Y i}}\end{array}\right]$ and $S_{2}=\left[\begin{array}{lll}I_{4} & 0_{4 \times 1} & 0_{4 \times q_{Y i}}\end{array}\right], z_{Y} \in R^{r_{Y} N}$ is the accessible output vector in order to verify the integrated FE/FTC performance with the matrices $\bar{C}_{x Y} \in R^{r_{Y} N \times 5 N}$ and $\bar{C}_{e Y} \in R^{r_{Y} N \times\left(6+q_{Y i}\right) N}$. Hence, the objective of the proposed integrated FE/FTC design is to devise the state feedback gain $K_{x Y}$, the distributed gain $K_{g Y}$, and the unknown input observer gains $H_{Y}$ and $J_{1 Y}$ to guarantee the robust stability of the integrated structure system (35).

Theorem 4.3: Given positive scalars $\gamma_{3}, \varepsilon_{Y 2}$ and $\varepsilon_{Y 3}$, matrices $\bar{C}_{x Y 0} \in R^{r_{Y} \times 5}, \bar{C}_{e Y x} \in R^{r_{Y} \times 5}, \bar{C}_{e Y a} \in R^{r_{Y} \times 1}$ and $\bar{C}_{e Y s} \in R^{r_{Y} \times q_{Y i}}$, the integrated FE/FTC system (35) is stable with the $H_{\infty}$ performance $\left\|G_{z_{Y} \bar{d}_{Y}}\right\|<\gamma_{3}$, if there exist symmetric positive-definite matrices $\bar{P}_{Y 1} \in R^{5 \times 5}, \bar{P}_{Y 2} \in R^{1 \times 1}, \bar{P}_{Y 3} \in R^{q_{Y i} \times q_{Y i}}$ and $\bar{Q}_{Y 0} \in R^{5 \times 5}$, and matrices $\bar{X}_{1} \in R^{1 \times 5}, \bar{X}_{2} \in R^{1 \times 3}, \bar{X}_{3} \in R^{5 \times 3}, \bar{X}_{4} \in R^{5 \times 3}, \bar{X}_{5} \in R^{1 \times 3}, \bar{X}_{6} \in R^{1 \times 3}, \bar{X}_{7} \in R^{q_{Y i} \times 3}$ and $\bar{X}_{8} \in R^{q_{Y i} \times 3}$ such that

$$
\left[\begin{array}{ccccccc}
\bar{\Omega}_{11} & \bar{\Omega}_{12} & \bar{\Omega}_{13} & \bar{\Omega}_{14} & 0 & 0 & \bar{\Omega}_{17} \\
\star & \bar{\Omega}_{22} & \bar{\Omega}_{23} & 0 & \bar{\Omega}_{25} & \bar{\Omega}_{26} & \bar{\Omega}_{27} \\
\star & \star & \bar{\Omega}_{33} & 0 & 0 & 0 & 0 \\
\star & \star & \star & \bar{\Omega}_{44} & 0 & 0 & 0 \\
\star & \star & \star & \star & \bar{\Omega}_{55} & 0 & 0 \\
\star & \star & \star & \star & \star & \bar{\Omega}_{66} & 0 \\
\star & \star & \star & \star & \star & \star & \bar{\Omega}_{77}
\end{array}\right]<0
$$

with

$$
\begin{gathered}
\bar{\Omega}_{22}=I_{N} \otimes\left[\begin{array}{ccc}
\bar{\Omega}_{211} & \bar{\Omega}_{212} & \bar{\Omega}_{213} \\
\star & \mathrm{He}\left(-\bar{X}_{5} C_{Y} B_{Y}\right) & \bar{\Omega}_{223} \\
\star & \star & \operatorname{He}\left(-\bar{X}_{8} F_{s Y}\right)
\end{array}\right], \bar{\Omega}_{23}=I_{N} \otimes\left[\begin{array}{ccc}
\bar{\Omega}_{2311} & 0 & -\bar{X}_{3} F_{s Y} \\
-\bar{X}_{5} C_{Y} D_{Y} & \bar{P}_{Y 2} & -\bar{X}_{5} F_{s Y} \\
-\bar{X}_{7} C_{Y} D_{Y} & 0 & \bar{P}_{Y 3}-\bar{X}_{7} F_{s Y}
\end{array}\right] \\
\bar{\Omega}_{26}=I_{N} \otimes\left[\begin{array}{ccc}
\bar{P}_{Y 1}-\bar{X}_{3} C_{Y} & 0 & -\bar{X}_{3} F_{s Y} \\
-\bar{X}_{5} C_{Y} & \bar{P}_{Y 2} & -\bar{X}_{5} F_{s Y} \\
-\bar{X}_{7} C_{Y} & 0 & \bar{P}_{Y 3}-\bar{X}_{7} F_{s Y}
\end{array}\right] \\
\bar{\Omega}_{211}=\operatorname{He}\left(\bar{P}_{Y 1} A_{Y}-\bar{X}_{3} C_{Y} A_{Y}-\bar{X}_{4} C_{Y}\right), \bar{\Omega}_{212}=\bar{P}_{Y 1} B_{Y}-\bar{X}_{3} C_{Y} B_{Y}-A_{Y}^{T} C_{Y}^{T} \bar{X}_{5}^{T}-C_{Y}^{T} \bar{X}_{6}^{T}, \\
\bar{\Omega}_{213}=-\bar{X}_{4} F_{s Y}-A_{Y}^{T} C_{Y}^{T} \bar{X}_{7}^{T}-C_{Y}^{T} \bar{X}_{8}^{T}, \bar{\Omega}_{223}=-\bar{X}_{6} F_{s Y}-B_{Y}^{T} C_{Y}^{T} \bar{X}_{7}^{T}, \bar{\Omega}_{2311}=\bar{P}_{Y 1} D_{Y}-\bar{X}_{3} C_{Y} D_{Y}, \\
\bar{\Omega}_{11}=I_{N} \otimes \operatorname{He}\left(\bar{Q}_{Y 0} A_{Y}-B_{Y} \bar{X}_{1}\right)-\mathcal{L} \otimes \operatorname{He}\left(g_{Y} B_{Y} \bar{X}_{2} C_{Y}\right)+\varepsilon_{Y 2} L_{g}^{2} I_{5 N}, \\
\bar{\Omega}_{12}=I_{N} \otimes\left[B_{Y} \bar{X}_{1} B_{Y} \hat{Q}_{Y 0} 0\right]-\mathcal{L} \otimes\left(g_{Y} B_{Y} \bar{X}_{2} F_{s Y} S_{1}\right), \bar{\Omega}_{13}=I_{N} \otimes\left(\bar{Q}_{Y 0} D_{Y} S_{2}\right), \\
\bar{\Omega}_{14}=I_{N} \otimes \bar{Q}_{Y 0}, \bar{\Omega}_{17}=I_{N} \otimes \bar{C}_{x Y 0}^{T}, \bar{\Omega}_{25}=I_{N} \otimes L_{g} A_{0}^{T}, \bar{\Omega}_{27}=I_{N} \otimes\left[\bar{C}_{e Y x} \bar{C}_{e Y a} \bar{C}_{e Y s}\right]^{T}, \\
\bar{\Omega}_{33}=-\gamma_{3}^{2} I_{\left(5+q_{Y i}\right) N}, \bar{\Omega}_{44}=-\varepsilon_{Y 2} I_{5 N}, \bar{\Omega}_{77}=-I_{r_{Y} N}, \bar{\Omega}_{55}=-\varepsilon_{Y 3}^{-1} I_{10}, \bar{\Omega}_{66}=-\varepsilon_{Y 3} I_{\left(6+q_{Y i}\right) N}
\end{gathered}
$$


Then, the designed gains for the integrated system are given by $K_{x Y}=\hat{\bar{Q}}_{Y 0}^{-1} \bar{X}_{1}, K_{g Y}=\hat{\bar{Q}}_{Y 0}^{-1} \bar{X}_{2}, H_{Y 1}=$ $\bar{P}_{Y 1}^{-1} \bar{X}_{3}, J_{1 Y 1}=\bar{P}_{Y 1}^{-1} \bar{X}_{4}, H_{Y 2}=\bar{P}_{Y 2}^{-1} \bar{X}_{5}, J_{1 Y 2}=\bar{P}_{Y 2}^{-1} \bar{X}_{6}, H_{Y 3}=\bar{P}_{Y 3}^{-1} \bar{X}_{7}$, and $J_{1 Y 3}=\bar{P}_{Y 3}^{-1} \bar{X}_{8}$ with $\bar{Q}_{Y 0} B_{Y}=$ $B_{Y} \hat{\bar{Q}}_{Y 0}$.

Proof: Consider the respective Lyapunov functions $V_{x_{Y}}=x_{Y}^{T} \bar{Q}_{Y} x_{Y}$ and $V_{e_{Y}}=e_{Y}^{T} \bar{P}_{Y} e_{Y}$ with symmetric positive-definite matrices $\bar{Q}_{Y}$ and $\bar{P}_{Y}$. Then, the respective time derivatives of $V_{x_{Y}}$ and $V_{e_{Y}}$ are obtained with positive scalar $\varepsilon_{Y 2}$ and $\varepsilon_{Y 3}$.

$$
\begin{aligned}
\dot{V}_{x_{Y}} & \leq x_{Y}^{T} \operatorname{He}\left(\bar{Q}_{Y}\left(I_{N} \otimes\left(A_{Y}-B_{Y} K_{x Y}\right)-g_{Y} \mathcal{L} \otimes B_{Y} K_{g Y} C_{Y}\right)\right) x_{Y}+\operatorname{He}\left(x_{Y}^{T} \bar{Q}_{Y}\left(I_{N} \otimes D_{Y} S_{2}\right) \bar{d}_{Y}\right) \\
& +\varepsilon_{Y 2}^{-1} x_{Y}^{T} \bar{Q}_{Y} \bar{Q}_{Y}^{T} x_{Y}+\varepsilon_{Y 2} g^{T}\left(x_{Y}\right) g\left(x_{Y}\right)+\operatorname{He}\left(x_{Y}^{T} \bar{Q}_{Y}\left(I_{N} \otimes B_{Y} K_{Y}-g_{Y} \mathcal{L} \otimes B_{Y} K_{g Y} F_{s Y} S_{1}\right) e_{Y}\right) \\
\dot{V}_{e Y} & \leq e_{Y}^{T}\left(\operatorname{He}\left(\bar{P}_{Y}\left(I_{N} \otimes\left(\Gamma_{Y} \bar{A}_{Y}-J_{1 Y} \bar{C}_{Y}\right)\right)\right)+\varepsilon_{Y 3} L_{g}^{2}\left(I_{N} \otimes A_{0}^{T} A_{0}\right)+\varepsilon_{Y 3}^{-1} \bar{P}_{Y}\left(I_{N} \otimes \Gamma_{Y} \Gamma_{Y}^{T}\right) \bar{P}_{Y}^{T}\right) e_{Y} \\
& +\operatorname{He}\left(e_{Y}^{T} \bar{P}_{Y}\left(I_{N} \otimes \Gamma_{Y} \bar{D}_{Y}\right) \bar{d}_{Y}\right)
\end{aligned}
$$

Denote $\bar{P}_{Y}=I_{N} \otimes \operatorname{diag}\left\{\bar{P}_{Y 1} \bar{P}_{Y 2} \bar{P}_{Y 3}\right\}, \bar{Q}_{Y}=I_{N} \otimes \bar{Q}_{Y 0}, \bar{C}_{x Y}=I_{N} \otimes \bar{C}_{x Y 0}$, and $\bar{C}_{e Y}=I_{N} \otimes\left[\bar{C}_{e Y x} \bar{C}_{e Y a} \bar{C}_{e Y s}\right]$ with symmetric positive-definite matrices $\bar{P}_{Y 1}, \bar{P}_{Y 2}, \bar{P}_{Y 3}$ and $\bar{Q}_{Y 0}$. Define matrices $H_{Y}=\left[H_{Y 1}^{T} H_{Y 2}^{T} H_{Y 3}^{T}\right]^{T}$ and $J_{1 Y}=\left[\begin{array}{lll}J_{1 Y 1}^{T} & J_{1 Y 2}^{T} & J_{1 Y 3}^{T}\end{array}\right]^{T}$. According to Definition 2.1, the sufficient condition of achieving $\left\|G_{z_{Y} \bar{d}_{Y}}\right\|<\gamma_{3}$ is $z_{Y}^{T} z_{Y}-\gamma_{3}^{2} \bar{d}_{Y}^{T} \bar{d}_{Y}+\dot{V}_{e Y}+\dot{V}_{x Y}<0$. Thus, the Schur Lemma is applied and the proof of Theorem 4.3 is straightforward and is omitted here.

Remark 4.2: Note that the undirected graph plays a role in the description of the LMI formulations, i.e., $\operatorname{He}\left(Q_{Y}\left(g_{Y} \mathcal{L} \otimes B_{Y} K_{g Y} C_{Y}\right)\right)$ in (31), and $\operatorname{He}\left(\bar{Q}_{Y}\left(g_{Y} \mathcal{L} \otimes B_{Y} K_{g Y} C_{Y}\right)\right)$ and $\operatorname{He}\left(\bar{Q}_{Y}\left(g_{Y} \mathcal{L} \otimes B_{Y} K_{g Y} F_{s Y} S_{1}\right)\right)$ in (37). Since the undirected graph $\mathcal{G}$ is connected, it follows from Lemma 2.1 that $x_{Y}^{T}\left(\mathcal{L} \otimes B_{Y} K_{g Y} C_{Y}\right) x_{Y} \geq$ $\lambda_{2} x_{Y}^{T}\left(I_{N} \otimes B_{Y} K_{g Y} C_{Y}\right) x_{Y}$ and $x_{Y}^{T}\left(\mathcal{L} \otimes B_{Y} K_{g Y} F_{s Y} S_{1}\right) x_{Y} \geq \lambda_{2} x_{Y}^{T}\left(I_{N} \otimes B_{Y} K_{g Y} F_{s Y} S_{1}\right) x_{Y}$, where $\lambda_{2}$ is the smallest nonzero eigenvalue of $\mathcal{L}$. In order to avoid the requirement of the global information of undirected graph, the following derivation is obtained.

$$
\begin{aligned}
\dot{V}_{x_{Y}} & \leq x_{Y}^{T} \operatorname{He}\left(\bar{Q}_{Y}\left(I_{N} \otimes\left(A_{Y}-B_{Y} K_{x Y}-\lambda_{2} g_{Y} B_{Y} K_{g Y} C_{Y}\right)\right)\right) x_{Y}+\operatorname{He}\left(x_{Y}^{T} \bar{Q}_{Y}\left(I_{N} \otimes D_{Y} S_{2}\right) \bar{d}_{Y}\right) \\
& +\varepsilon_{Y 2}^{-1} x_{Y}^{T} \bar{Q}_{Y} \bar{Q}_{Y}^{T} x_{Y}+\varepsilon_{Y 2} g^{T}\left(x_{Y}\right) g\left(x_{Y}\right)+\operatorname{He}\left(x_{Y}^{T} \bar{Q}_{Y}\left(I_{N} \otimes\left(B_{Y} K_{Y}-\lambda_{2} g_{Y} B_{Y} K_{g Y} F_{s Y} S_{1}\right)\right) e_{Y}\right)
\end{aligned}
$$

Remark 4.3: The general dynamics (18) in the $Y$ channel are selected in Theorems 4.1-4.3. Furthermore, the integrated FE/FTC model based on the augmented dynamics (17) in the $X$ channel is considered with available output information.

$$
\left\{\begin{aligned}
\dot{x}_{X}= & \left(I_{N} \otimes\left(A_{X}-B_{X} K_{x X}\right)-g_{X} \mathcal{L} \otimes B_{X} K_{g X} C_{X}\right) x_{X} \\
& +\left(I_{N} \times B_{X} K_{X}-g_{X} \mathcal{L} \otimes B_{X} K_{g X} F_{s X} S_{3}\right) e_{X}+\left(I_{N} \otimes D_{X} S_{4}\right) \bar{d}_{X} \\
\dot{e}_{X}= & \left(I_{N} \otimes\left(\Gamma_{X} \bar{A}_{X}-J_{1 X} \bar{C}_{X}\right)\right) e_{X}+\left(I_{N} \otimes \Gamma_{X} \bar{D}_{X}\right) \bar{d}_{X} \\
z_{X}= & \bar{C}_{x X} x_{X}+\bar{C}_{e X} e_{X}
\end{aligned}\right.
$$

where $S_{3}=\left[\begin{array}{llll}0_{q_{X i} \times 4} & 0_{q_{X i} \times 1} & I_{q_{X i}}\end{array}\right]$ and $S_{4}=\left[\begin{array}{lll}I_{5} & 0_{5 \times 1} & I_{5 \times q_{X i}}\end{array}\right]$. Note that only the estimation error $e_{X}$ from the FE process in the $X$ channel influences the FTC performance and the FTC process does not influence the FE performance in turn.

Remark 4.4: Note that the additive actuator fault $f_{h i}^{a}$ occurs in the $i$-th UAV and the actuator fault $f_{h(i-1)}^{a}$ occurs in the $(i-1)$-th UAV in the $Z$ channel. The existing fault $f_{h(i-1)}^{a}$ makes the distributed FTC controller $(27)$ 
not appropriate in the FTC process. Thus, the distributed fault-tolerant controller for the $i$-th UAV in the vertical direction is designed as

$$
u_{i Z}=-K_{Z} \hat{\bar{x}}_{i Z}-g_{Z} K_{g Z} \sum_{j=1}^{N} a_{i j}\left(y_{i Z}-y_{j Z}\right)
$$

where $K_{Z}=\bar{B}_{Z}^{\dagger} \bar{A}_{Z}$ with $\bar{B}_{Z}^{\dagger}=\left(\bar{B}_{Z}^{T} \bar{B}_{Z}\right)^{-1} \bar{B}_{Z}^{T}$. Furthermore, the integrated FE/FTC model in the $Z$ channel is considered with the distributed fault-tolerant controller (41).

$$
\left\{\begin{array}{l}
\dot{\bar{x}}_{Z}=\left(I_{N} \otimes \bar{A}_{Z}\right) e_{Z}-\left(g_{Z} \mathcal{L} \otimes \bar{B}_{Z} K_{g Z} \bar{C}_{Z}\right) \bar{x}_{Z}+\left(I_{N} \otimes \bar{D}_{Z}\right) \bar{d}_{Z} \\
\dot{e}_{Z}=\left(I_{N} \otimes\left(\Gamma_{Z} \bar{A}_{Z}-J_{1 Z} \bar{C}_{Z}\right)\right) e_{Z}+\left(I_{N} \otimes \Gamma_{Z} \bar{D}_{Z}\right) \bar{d}_{Z} \\
z_{Z}=\bar{C}_{x Z} \bar{x}_{Z}+\bar{C}_{e Z} e_{Z}
\end{array}\right.
$$

Note that only the estimation error $e_{Z}$ from the FE process in the $Z$ channel influences the FTC performance and the FTC process does not influence the FE performance in turn.

\section{Simulation Results}

In this section, an application of integrated FE and FTC scheme for CFF models with simultaneous actuator and sensor faults is put forward to validate the effectiveness of the proposed control scheme. The parameters of CFF models in the $X, Y$ and $Z$ channels are characterized in TABLE I. The simulated parameters of the FE/FTC schemes in Theorems 4.1-4.3 are designed as $\varepsilon_{Y 2}=\varepsilon_{Y 3}=g_{Y}=1, \gamma_{3}=0.1, \bar{C}_{x Y 0}=\bar{C}_{e Y x}=\left[\begin{array}{llll}-1 & 1.2 & 0.5 & 0.10\end{array}\right], \bar{C}_{e Y a}=$ $\bar{C}_{e Y s}=0$, and the sensor fault distribution matrices are satisfied with $F_{s X}=\left[\begin{array}{ll}1 & 0\end{array}\right]^{T}, F_{s Y}=\left[\begin{array}{lll}1 & 0 & 1\end{array}\right]^{T}$, and $F_{s Z}=1$. Then, the unknown input observer gains and the FE/FTC gains are derived as

TABLE I

THE PARAMETERS OF THE CFF MODEL[8], [23].

\begin{tabular}{|c|c|c|c|}
\hline Symbol & Value(unit) & Symbol & Value(unit) \\
\hline$S$ & $54.75(\mathrm{~m})$ & $\tau_{\psi a}, \tau_{\psi b}$ & $0.919(\mathrm{~s})$ \\
\hline$m$ & $16057(\mathrm{~kg})$ & $\tau_{v}$ & $5(\mathrm{~s})$ \\
\hline$A_{R}$ & 3 & $\tau_{h a}$ & $0.3075(\mathrm{~s})$ \\
\hline$S_{v t}$ & $27.87\left(\mathrm{~m}^{2}\right)$ & $\tau_{h b}$ & $3.85(\mathrm{~s})$ \\
\hline$h_{z}$ & $3.3(\mathrm{~m})$ & $v_{c}$ & $135(\mathrm{~m} / \mathrm{s})$ \\
\hline$a_{w}, a_{v t}$ & $5.3(1 / \mathrm{rad})$ & $\psi_{c}$ & $-8^{\circ}$ \\
\hline$C_{L_{l}}, C_{L_{w}}$ & 0.5385 & $h_{c}$ & $900(\mathrm{~m})$ \\
\hline$\eta$ & 0.95 & $x_{c}$ & $18.9(\mathrm{~m})$ \\
\hline$b$ & $9.45(\mathrm{~m})$ & $y_{c}$ & $7.42(\mathrm{~m})$ \\
\hline$q$ & $743.48(\mathrm{KPa})$ & $z_{c}$ & $0(\mathrm{~m})$ \\
\hline$p_{D_{w y}}$ & -0.000783 & $p_{L_{w y}}$ & 0.00771 \\
\hline$p_{S_{w y}}$ & -0.026 & $p_{L_{w z}}$ & -0.0702 \\
\hline
\end{tabular}




$$
\begin{aligned}
& K_{X}=\left[\begin{array}{llll}
-382.5806 & 209.7101 & 350.8470 & 70.8220
\end{array}\right], K_{Z}=\left[\begin{array}{lll}
34.4142 & 12.0474 & -28.7564
\end{array}\right] \\
& K_{x Y}=\left[\begin{array}{lllll}
-0.7936 & -5.2812 & -0.0943 & 3.6588 & 3.4755
\end{array}\right], K_{g Y}=\left[\begin{array}{lll}
-0.6887 & -0.1054 & 3.1247
\end{array}\right] \\
& G_{Y}=\left[\begin{array}{c}
0 \\
0 \\
0 \\
0 \\
0 \\
-0.1119 \\
0
\end{array}\right], H_{Y}=\left[\begin{array}{ccc}
0.0266 & -0.0266 & 0 \\
-0.0565 & 0.0565 & 0 \\
-0.5661 & 0.5661 & 0 \\
0.6785 & -0.6785 & 0 \\
-1.2357 & 0.2357 & 1 \\
-2.9467 & -1.2588 & 4.2055 \\
0.8615 & 0.1385 & 0
\end{array}\right], J_{Y}=\left[\begin{array}{ccc}
0.9811 & -0.1014 & -0.8797 \\
0.0217 & 0.0875 & -0.1092 \\
-0.3889 & 0.4821 & -0.0932 \\
-0.0123 & -1.2979 & 1.3101 \\
1.6721 & -0.2859 & -1.3862 \\
0.3296 & 0.2527 & -0.5823 \\
-0.5279 & -0.1356 & 0.6635
\end{array}\right] \\
& M_{Y}=\left[\begin{array}{ccccccc}
-0.8874 & 0 & 0.1494 & 0 & -0.2131 & 0 & -0.0714 \\
-0.9828 & 0 & -0.1515 & 0 & -0.0022 & 0 & -0.0274 \\
0.3154 & 0 & -1.0465 & 0 & 0.3334 & 0 & -0.3045 \\
-0.2065 & 0 & 0.8184 & 0 & 0.0265 & 0 & 0.3282 \\
0.4783 & 0 & -0.2982 & 0 & -1.6554 & 0 & -0.0892 \\
0 & 0 & 0 & 0 & 0 & -0.1119 & 0 \\
-0.2877 & 0 & -0.1515 & 0 & -0.1502 & 0 & -1.2529
\end{array}\right] \\
& \Gamma_{Y}=\left[\begin{array}{ccccccc}
0.9734 & 0 & 0.0266 & 0 & 0 & 0 & 0 \\
0.0565 & 1 & -0.0565 & 0 & 0 & 0 & 0 \\
0.5661 & 0 & 0.4339 & 0 & 0 & 0 & 0 \\
-0.6785 & 0 & 0.6785 & 1 & 0 & 0 & 0 \\
1.2357 & 0 & -0.2357 & 0 & 0 & 0 & 0 \\
2.9467 & 0 & 1.2588 & 0 & -4.2055 & 1 & 0 \\
-0.8615 & 0 & -0.1385 & 0 & 0 & 0 & 0
\end{array}\right]
\end{aligned}
$$

To demonstrate the effectiveness of the proposed separated and integrated FE/FTC designs in Theorems 4.1-4.3, the maneuver step inputs and the actuator and sensor faults per combined maneuvering case are described in TABLE II. The table shows that the same velocity, heading angle and altitude maneuvers are applied to two cases, in which the fault-free case is considered in Case 1, the actuator faults are injected into the $X$ and $Z$ channels and the simultaneous actuator and sensor faults are considered in the $Y$ channel in Case 2.

As can be seen in Figures 2 and 3 of Case 1, the velocities and altitudes of the Lead and Wing UAVs are always coincident whilst the Lead UAV is being maneuvered at the respective $t=10 \mathrm{~s}$ and $t=30 \mathrm{~s}$ in combined form. The separations in the $X$ and $Z$ channels vary and finally remain at rated values due to the lack of prophetic information of maneuvering, thus, resulting in the time delay of tracking. Figure 2 of Case 1 shows that the heading angles of the Wing UAVs track with that of the Lead UAV while the Lead UAV is being maneuvered at $t=20 \mathrm{~s}$. The separations in the $X$ and $Y$ channels increase first and then decrease at rated values because the Wing UAVs on the outside need to fly farther away to track with the Lead UAV in order to keep the formation geometry.

Figure 4 of Case 2 shows that the velocities of the Wing UAVs track with that of the Lead UAV whilst the 

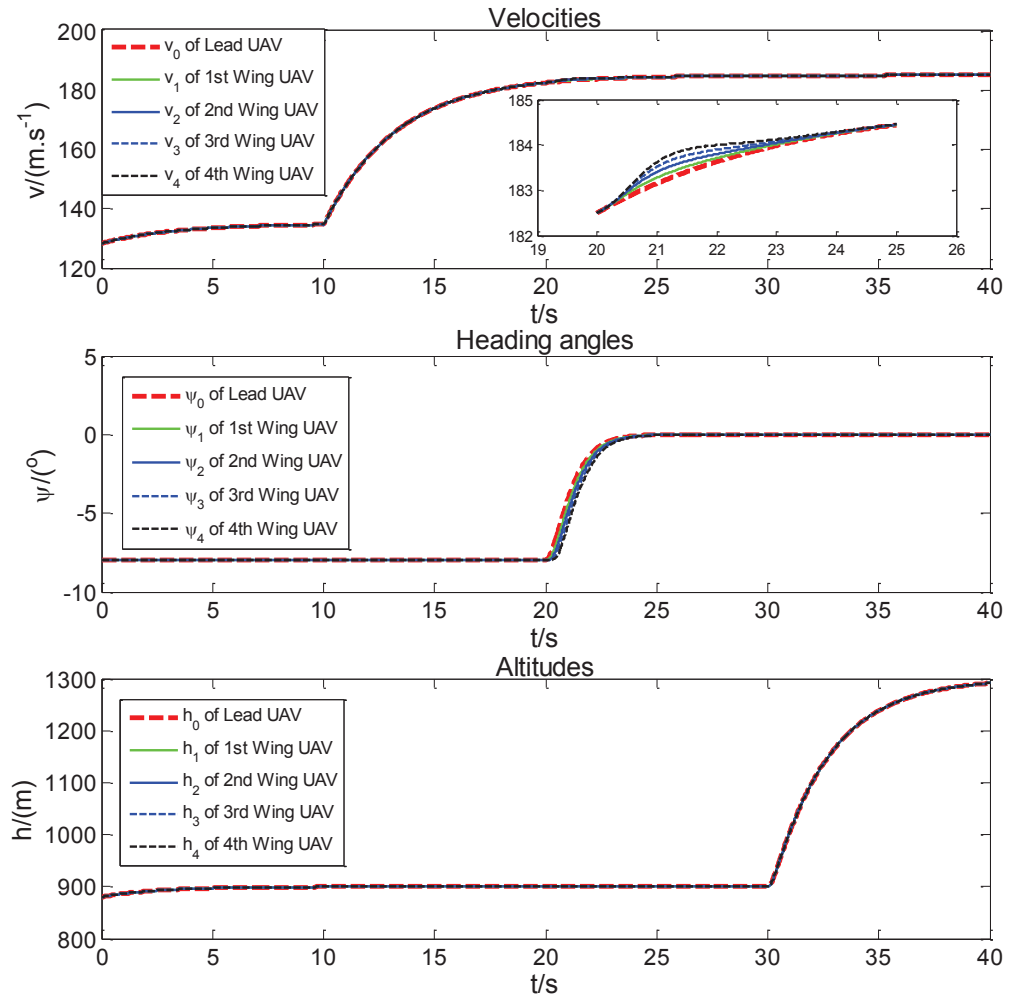

Fig. 2. Case 1: the combined maneuvering without actuator/sensor faults.
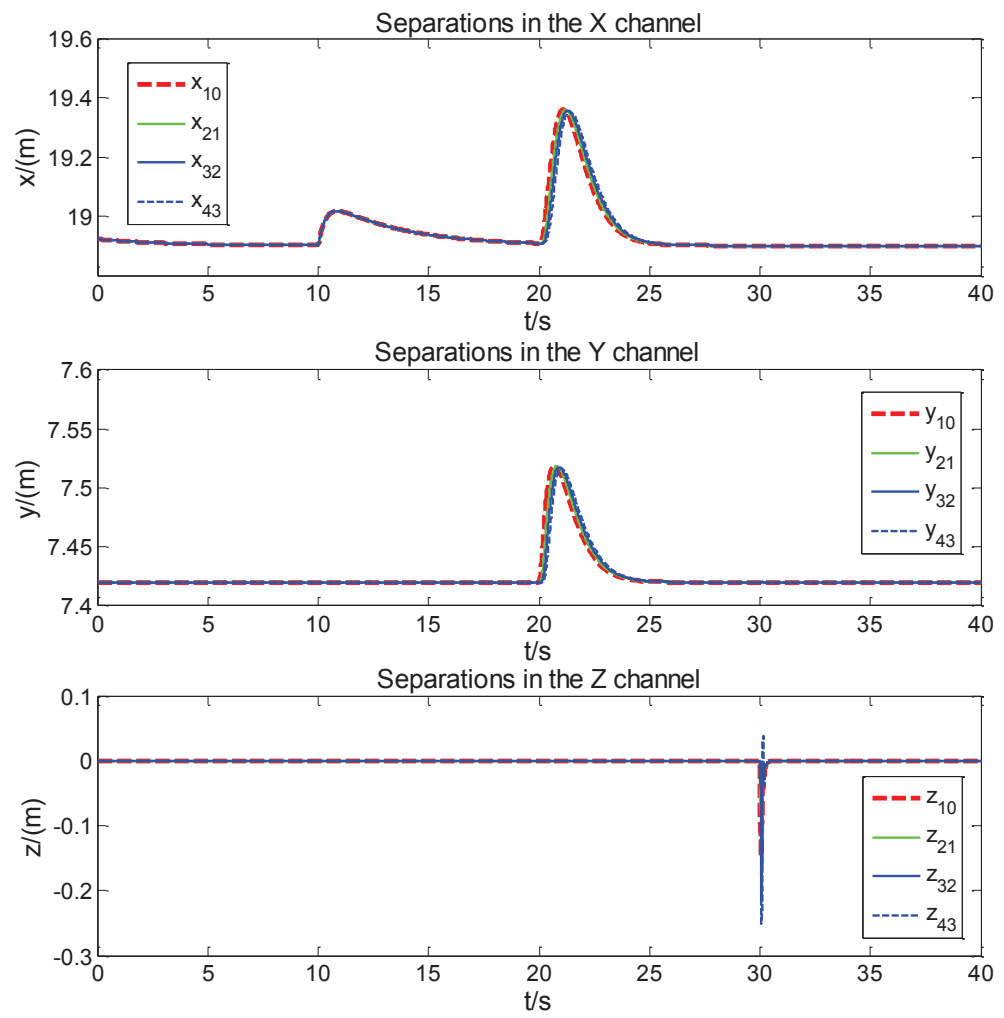

Fig. 3. Case 1: the combined maneuvering without actuator/sensor faults. 
TABLE II

MANEUVER STEP INPUTS AND THE ACTUATOR AND SENSOR FAULTS PER COMBINED MANEUVERING CASE.

\begin{tabular}{|c|c|c|c|}
\hline Maneuvering & Velocity & Heading angle & Altitude \\
\hline Case 1 & $+50 \mathrm{~m} / \mathrm{s}, \mathrm{t}=10 \mathrm{~s}$ & $+8^{\circ}, \mathrm{t}=20 \mathrm{~s}$ & $+400 \mathrm{~m}, \mathrm{t}=30 \mathrm{~s}$ \\
\hline Case 2 & $+50 \mathrm{~m} / \mathrm{s}, \mathrm{t}=10 \mathrm{~s}$ & $+8^{\circ}, \mathrm{t}=20 \mathrm{~s}$ & $+400 \mathrm{~m}, \mathrm{t}=30 \mathrm{~s}$ \\
\hline Fault & Velocity & Heading angle & Altitude \\
\hline Case 1 & $\times$ & $\times$ & $\times$ \\
\hline Case 2 & $f_{v 1}^{a}=-30 \mathrm{~m} / \mathrm{s}, \mathrm{t}=15 \mathrm{~s}$ & $f_{\psi 1}^{a}=+8^{\circ}, \mathrm{t}=5 \mathrm{~s}$ & $f_{h 1}^{a}=+30 \mathrm{~m}$, \\
& $f_{v 1}^{a}=-30 \mathrm{~m} / \mathrm{s}, \mathrm{t}=25 \mathrm{~s}$ & $f_{\psi 1}^{a}=+8^{\circ}, \mathrm{t}=35 \mathrm{~s}$ & $\mathrm{t}=30 \mathrm{~s}$ \\
& & $f_{1 Y}^{s}=-5 \mathrm{~m}, \mathrm{t}=5 \mathrm{~s}$ & \\
& & $f_{1 Y}^{s}=-5 \mathrm{~m}, \mathrm{t}=35 \mathrm{~s}$ & \\
\hline
\end{tabular}

velocity of the Lead UAV is being maneuvered at $t=10 \mathrm{~s}$ under the actuator faults in the respective time constants $t=15 \mathrm{~s}$ and $25 \mathrm{~s}$. Figure 5 of Case 2 shows that the heading angles of the Wing UAVs track with that of the Lead UAV whilst the heading angle of the Lead UAV is being maneuvered at $t=20$ s under simultaneous actuator and sensor faults in the respective time constants $t=5 \mathrm{~s}$ and 35s. Figure 6 of Case 2 shows that the altitude curves of the Lead and Wing UAVs are coincident whilst the altitude of the Lead UAV is being maneuvered at $t=30$ s under the actuator faults at the time constant $t=30$ s. Meanwhile, all the separation curves in the $X, Y$ and $Z$ channels amongst each UAV finally remain at rated values and show small amplitudes of the oscillations in the convergence process at each fault-occurring time instance. Furthermore, the velocity maneuvering does not affect the $Y$ and $Z$ channels while the altitude maneuvering affects the $Y$ channel due to the coupling item $\frac{q S}{m} p_{S_{w z}}$. Furthermore, there is a small oscillation on the separation in the $Z$ channel due to the coupling item $\frac{q S}{m} p_{L_{w y}}$ between the $Y$ and $Z$ channels.
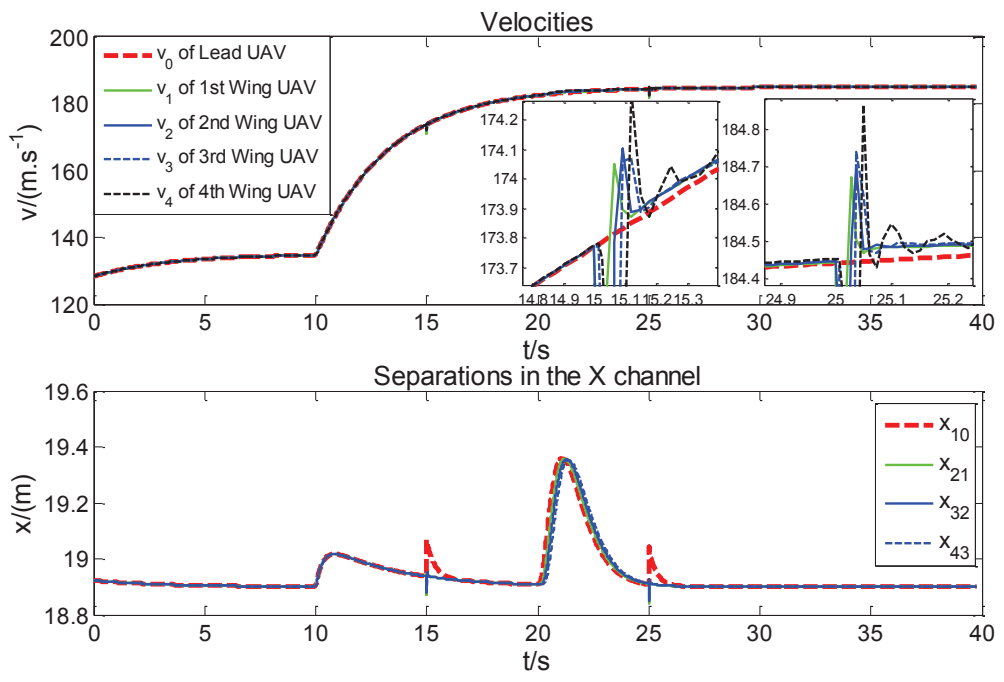

Fig. 4. Case 2 with simultaneous actuator/sensor faults ( $X$ channel). 

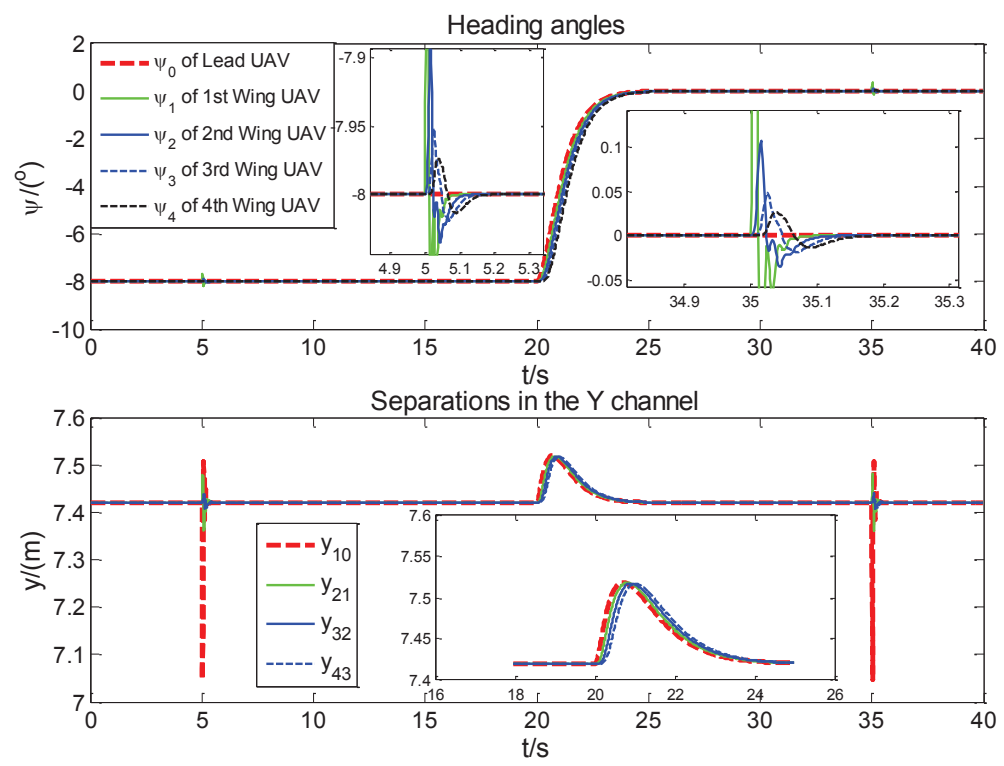

Fig. 5. Case 2 with simultaneous actuator/sensor faults ( $Y$ channel).
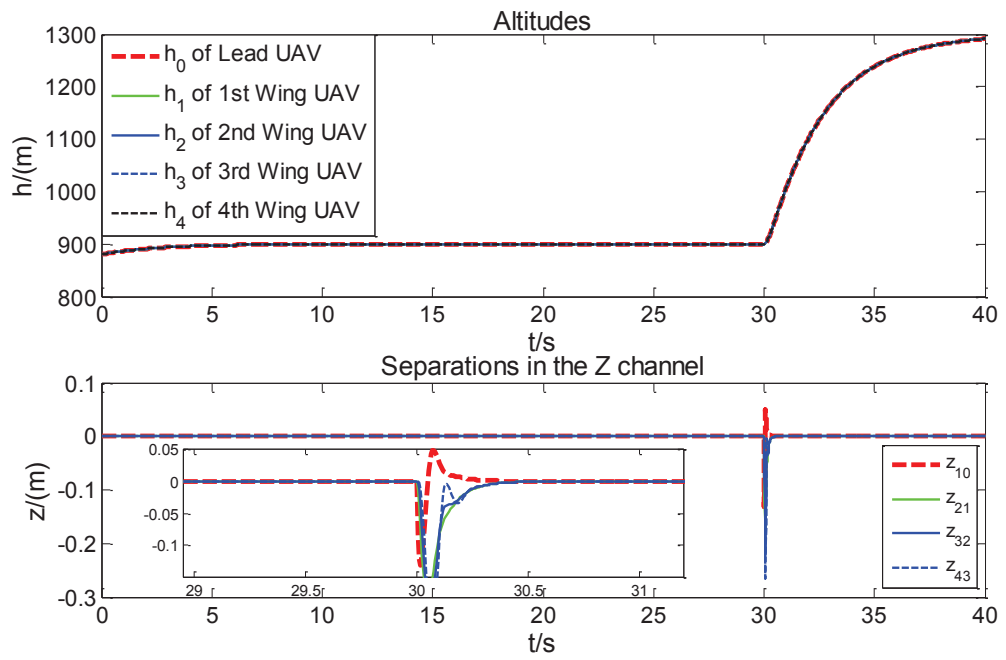

Fig. 6. Case 2 with simultaneous actuator/sensor faults $(Z$ channel).

The curves in Figures 7 and 8 of Case 2 simulated by both the approach[23] and the proposed integrated FE/FTC algorithm show the good tracking properties of rated and estimated actuator and sensor faults in the respective $X, Y$ and $Z$ channels. Compared with our previous study[23], although there exists a sharp peak in the proposed integrated FE/FTC scheme due to its rapid convergence, the integrated algorithm shows faster convergence and smaller amplitudes of the oscillations in estimated faults to an extent.

Figures 9 and 10 show that the heading angles of the four Wing UAVs track with the rated angle of the Lead UAV, i.e., $\left(\psi_{0}=-8^{\circ}\right)$ at $t=5 \mathrm{~s}$ and $\left(\psi_{0}=0^{\circ}\right)$ at $t=35 \mathrm{~s}$. The separations amongst each $\mathrm{UAV}$ remain to the rated values, i.e., $\left(x_{c}=18.9 \mathrm{~m}\right)$ in the $X$ channel in Figure 11 and $\left(y_{c}=7.42 \mathrm{~m}\right)$ in the $Y$ channel in Figure 12. Due to the 

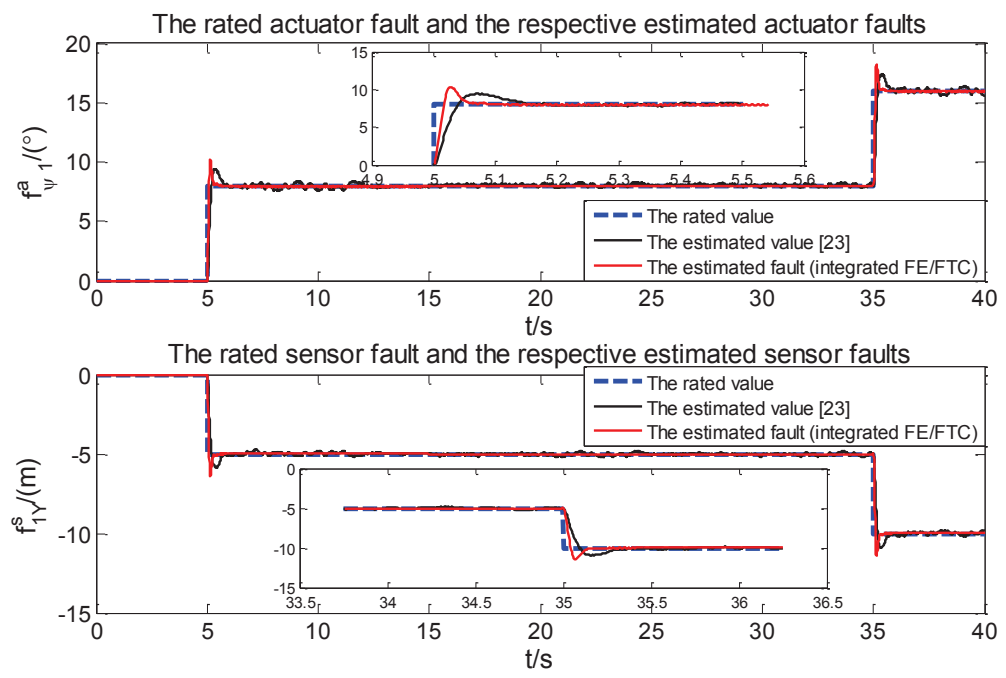

Fig. 7. Case 2: the respective estimated actuator and sensor faults in the $Y$ channel with the integrated FE/FTC scheme and the control scheme in [23].
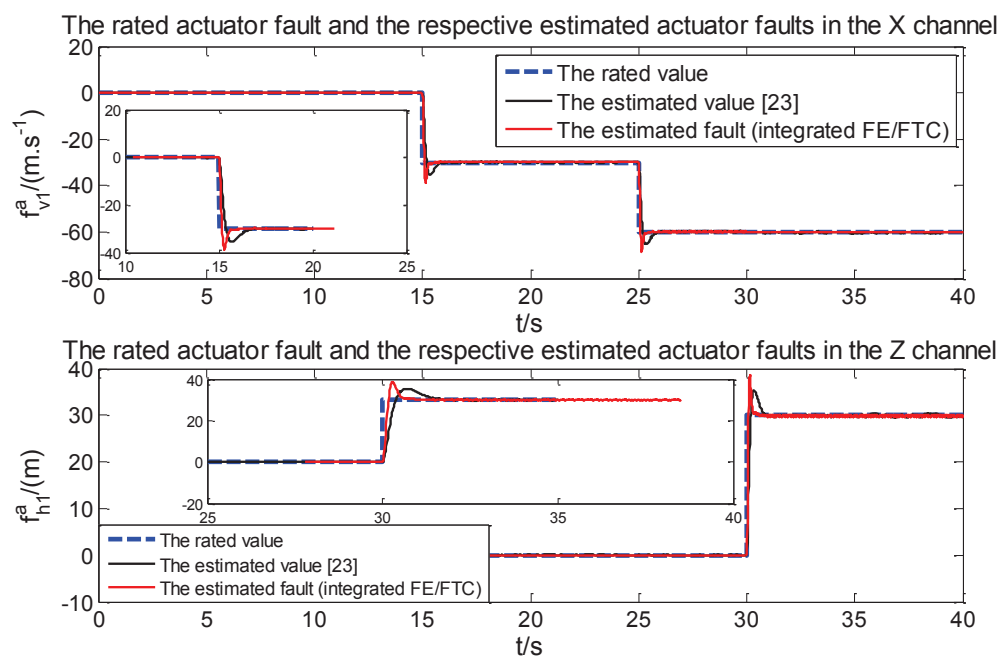

Fig. 8. Case 2: the respective estimated actuator faults in the $X$ and $Z$ channels with the integrated FE/FTC scheme and the control scheme in [23].

existence of the coupling item $\frac{q S}{m} p_{D_{w y}}$ and the parameter selection of $C_{x X}$ and $C_{e X}$ in the separated design, more oscillation responses are shown in the separated FE/FTC in Figure 11. Compared with the separated FE/FTC in Theorems 4.1 and 4.2, the integrated FE/FTC in Theorem 4.3 shows a smaller convergence amplitude of the heading angles and separations in the $X$ and $Y$ channels at each fault occurring time instant because the integrated FTC system contains more information from the FE process. Figure 13 shows the position-space trajectories of each Wing UAVs for two different Lead UAV maneuvers (with actuator/sensor faults from Case 2). All the trajectories show some separation errors when the actuator or sensor faults occur, but quickly return to the rated values. Hence, the combined maneuvering cases with simultaneous actuator/sensor faults demonstrate the effectiveness of the proposed 
integrated FE/FTC control scheme for CFF systems, and the control objective is achieved.

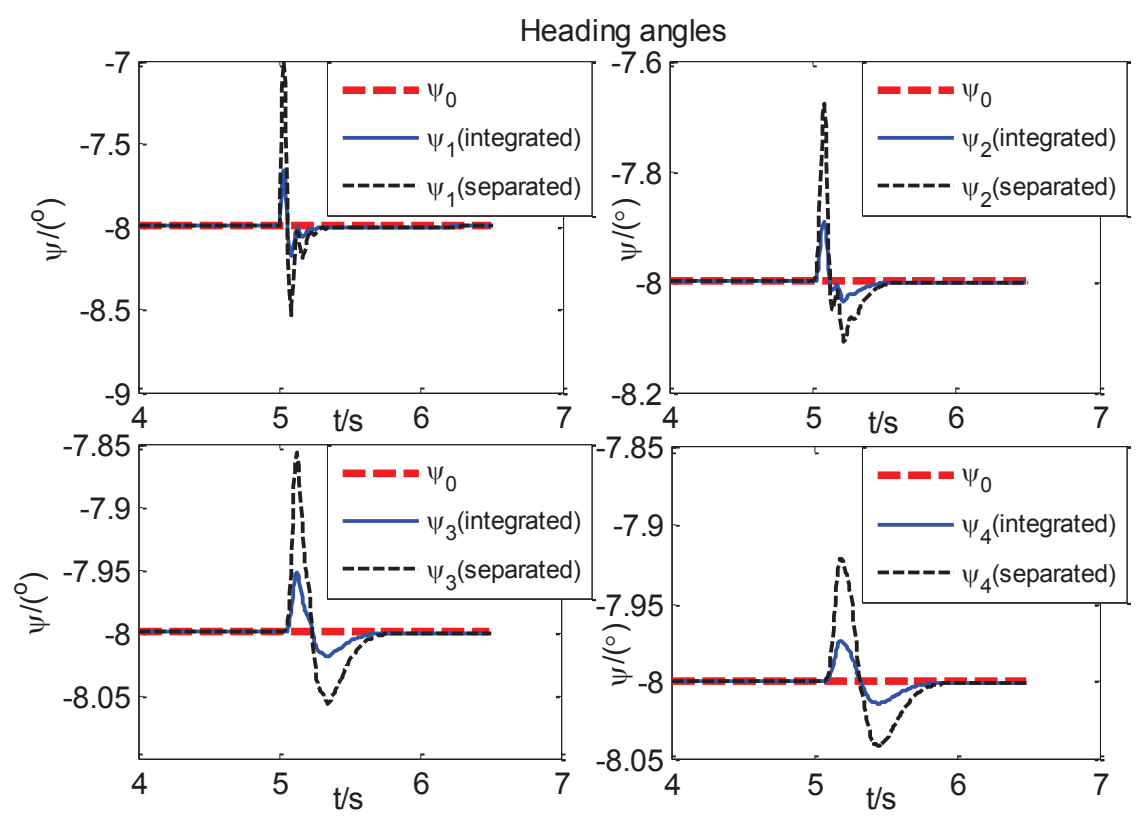

Fig. 9. Case 2: the heading angles at $t=5 \mathrm{~s}$ with the separated and integrated FE/FTC schemes ( $Y$ channel).
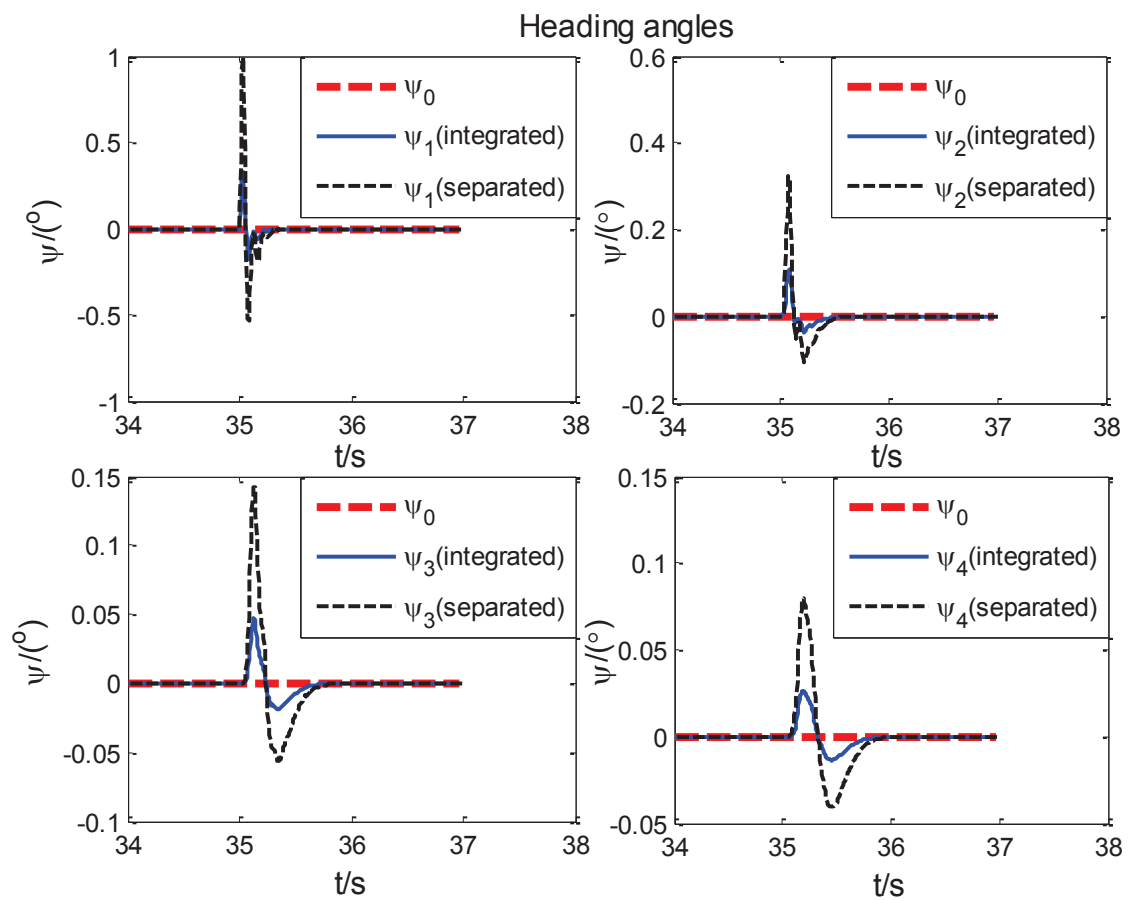

Fig. 10. Case 2: the heading angles at $t=35 \mathrm{~s}$ with the separated and integrated FE/FTC schemes ( $Y$ channel). 


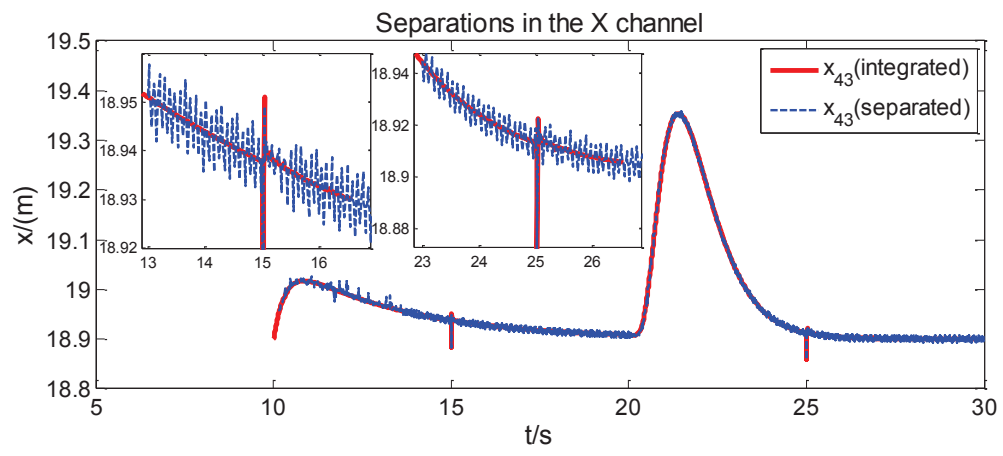

Fig. 11. Case 2 with separated and integrated FE/FTC schemes ( $X$ channel).
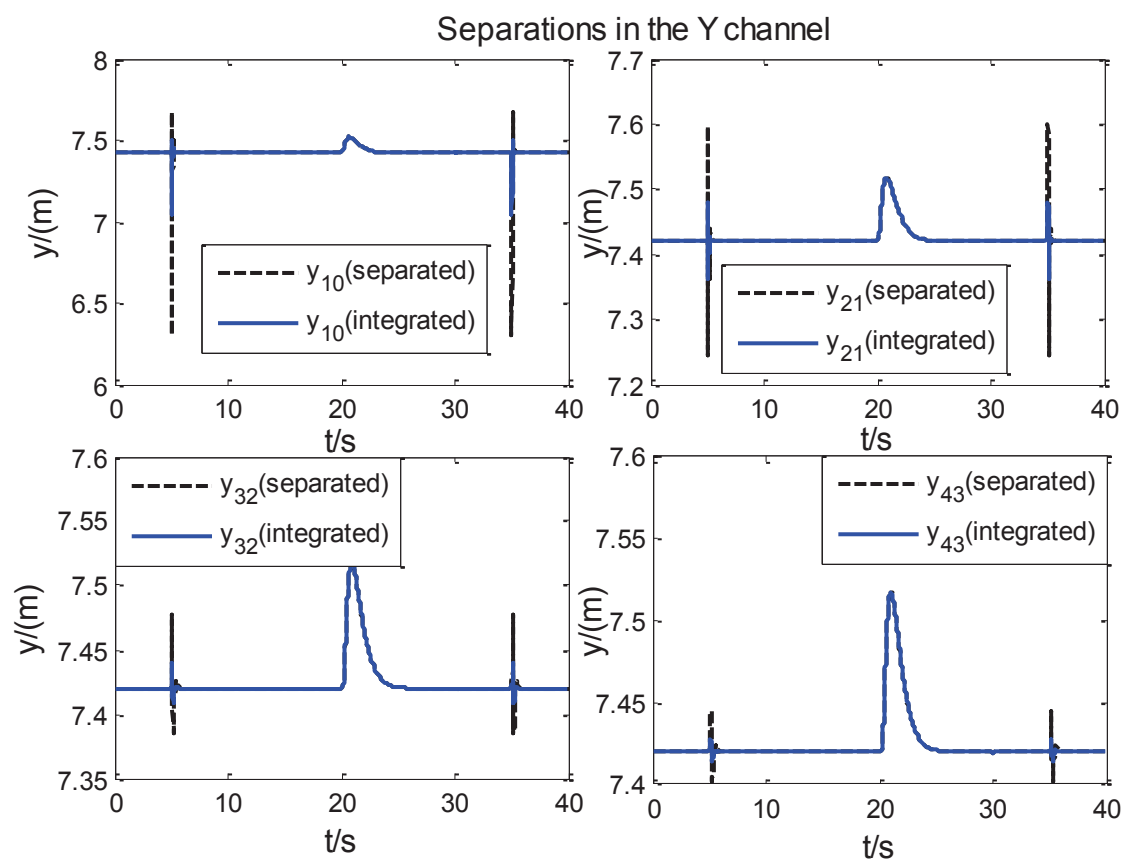

Fig. 12. Case 2 with separated and integrated FE/FTC schemes ( $Y$ channel).

\section{CONCLUSION}

In this study, an integrated FE and FTC design has been developed for CFF systems with simultaneous actuator and sensor faults to track the position and attitude of commanded motions of the Lead UAV. Compared with the separated design, integrated FE/FTC design considers the bidirectional interactions between FE and FTC systems and makes full use of the estimated fault information of FE system, so that the convergence amplitude in the integrated design is smaller. Integrated designs, namely, decentralized FE and distributed FTC protocols, are proposed to guarantee the excellent tracking of position and attitude whilst the Lead UAV is being maneuvered. Current investigations focus on extensions of the proposed method to unpredicted maneuvering, communication faults, mission completion and formation reconfiguration. 
Geodetic coordinate system in 3-D space
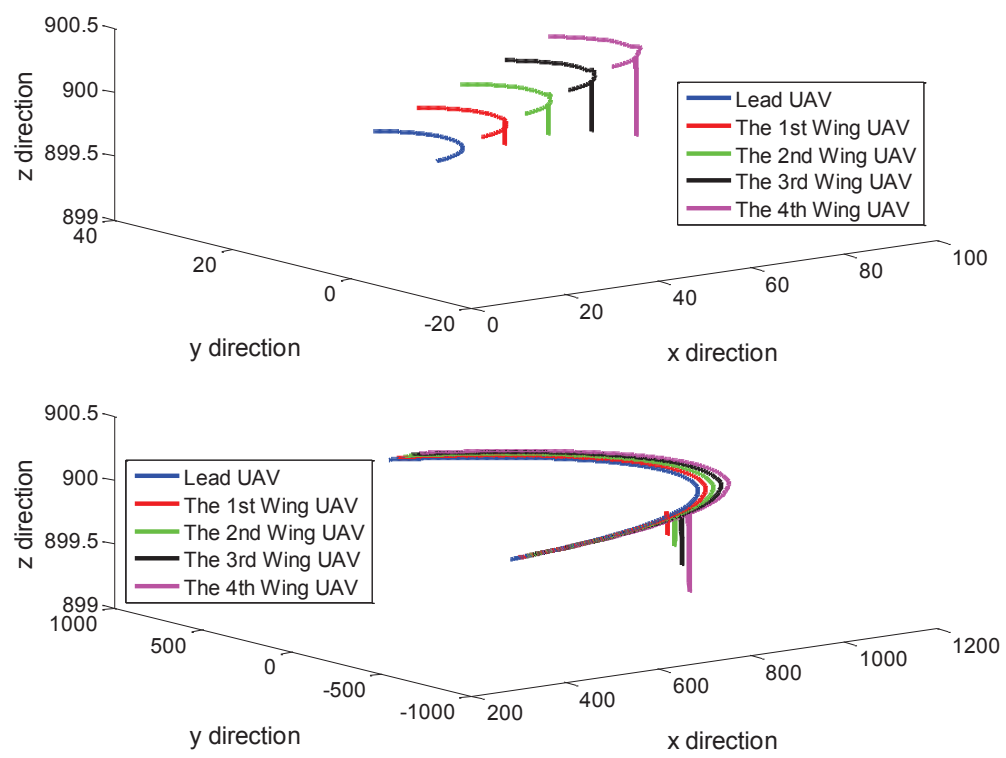

Fig. 13. Case 2: UAV trajectories in 3-D space.

\section{REFERENCES}

[1] Zhang, Q. R. and Liu, H. T., “Aerodynamics modeling and analysis of close formation flight,” J. Aircr., vol. 54, no. 6, pp. 2192-2204, Jul. 2017.

[2] Shan, J. J. and Liu, H. T., "Close-formation flight control with motion synchronization," J. Guid. Control Dyn., vol. 28, no. 6, pp. 1316-1320, Nov. 2005.

[3] Bangash, Z. A., Sanchez, R. P., Ahmed, A., and Khan, M. J., “Aerodynamics of formation flight," J. Aircr., vol. 43, no. 4, pp. 907-912, Jul. 2006.

[4] Cho, H., Lee, S., and Han, C., "Experimental study on the aerodynamic characteristics of a fighter-type aircraft model in close formation flight," J. Mech. Sci. Technol., vol. 28, no. 8, pp. 3059-3065, Aug. 2014.

[5] Singh, S. N., Chandler, P., Schumacher, C., Banda, S., and Pachter, M., "Nonlinear adaptive close formation control of unmanned aerial vehicles," Dyn. Control, vol. 10, no. 2, pp. 179-194, Apr. 2000.

[6] Singh, S. N., Pachter, M., Chandler, P., Banda, S., Rasmussen, S., and Schumacher, C., "Input-output invertibility and sliding mode control for close formation flying of multiple UAVs,” Int. J. Robust Nonlinear Control, vol. 10, pp. 779-797, Jul. 2000.

[7] Qiu, H. X. and Duan, H. B., "Receding horizon control for multiple UAV formation flight based on modified brain storm optimization," Nonlinear Dyn., vol. 78, no. 3, pp. 1973-1988, Nov. 2014.

[8] Pachter, M., D’Azzo, J. J., and Proud, A. W., “Tight formation flight control,” J. Guid. Control Dyn., vol. 24, no. 2, pp. $246-254$, Apr. 2001.

[9] Singh, S. N., Zhang, R., Chandler, P., and Banda, S., "Decentralized nonlinear robust control of UAVs in close formation," Int. J. Robust Nonlinear Control, vol. 13, pp. 1057-1078, Mar. 2003.

[10] Qiu, H. X. and Duan, H. B., "Multiple UAV distributed close formation control based on in-flight leadership hierarchies of pigeon flocks," Aerosp. Sci. Technol., vol. 70, pp. 471-486, Nov. 2017.

[11] Johnson, Y. and Dasgupta, S., "Control and tracking of roll dynamics of UAVs in close formation flight," in Signal Process. Inf. Commun. Energ. Syst. Conf., 2015, pp. 1-5.

[12] DInnocenzo, A., Benedtetto, M. D., and Serra, E., "Fault tolerant control of multi-hop control networks," IEEE Trans. Autom. Control, vol. 58, no. 6, pp. 1377-1389, Jun. 2013.

[13] Izadi, H. A., Gordon, B. W., and Zhang, Y. M., "Hierarchical decentralized receding horizon control of multiple vehicles with communication failures," IEEE Trans. Aerosp. Electron. Syst., vol. 49, no. 2, pp. 744-759, Apr. 2013. 
[14] Chen, G., Song, Y. D., and Lewis, F. L., "Distributed fault-tolerant control of networked uncertain Euler-Lagrange systems under actuator faults," IEEE Trans. Cybern., vol. 47, no. 7, pp. 1706-1718, Jul. 2017.

[15] Yu, X., Liu, Z. X., and Zhang, Y. M., "Fault-tolerant formation control of multiple UAVs in the presence of actuator faults," Int. J. Robust Nonlinear Control, vol. 26, no. 12, pp. 2668-2685, Aug. 2016.

[16] Xu, Q., Yang, H., Jiang, B., and Zhang, Y. M., "Fault tolerant formations control of UAVs subject to permanent and intermittent faults," Int. Intell. Rob. Syst., vol. 73, pp. 589-602, Jan. 2014.

[17] Hua, Y. Z., Dong, X. W., Li, Q. D., and Zhang, R., "Distributed fault-tolerant time-varying formation control for high-order linear multiagent systems with actuator failures," ISA Trans., vol. 71, no. 1, pp. 40-50, Nov. 2017.

[18] Hu, Q. L., Wang, C. L., Li, Y., and Huang, J., "Adaptive control for hypersonic vehicles with time-varying faults," IEEE Trans. Aerosp. Electron. Syst., vol. 54, no. 3, pp. 1442-1455, Jun. 2018.

[19] Ma, H. J. and Yang, G. H., "Adaptive fault tolerant control of cooperative heterogeneous systems with actuator faults and unreliable interconnections," IEEE Trans. Autom. Control, vol. 61, no.11, pp. 3240-3255, Nov. 2016.

[20] Qin, L. G., He, X., and Zhou, D. H., "Fault-tolerant cooperative output regulation for multi-vehicle systems with sensor faults," Int. J. Control, vol. 90, no. 10, pp. 2227-2248, 2017.

[21] Zhu, J. W., Yang, G. H., Zhang, W. A., and Yu, L., "Cooperative fault tolerant tracking control for multiagent systems: an intermediate estimator-based approach,” IEEE Trans. Cybern., vol. 48, no. 10, pp. 2972-2980, Oct. 2018.

[22] Hu, Q. L., Zhang, X. D., and Chen, W. H., "Robust fault-tolerant tracking control for spacecraft proximity operations using time-varying sliding mode," IEEE Trans. Aerosp. Electron. Syst., vol. 54, no. 1, pp. 2-17, Feb. 2018.

[23] Liu, C., Jiang, B., and Zhang, K., "Integrated multiple-model adaptive fault identification and reconfigurable fault tolerant control for Lead-Wing close formation systems," Int. J. Syst. Sci., vol. 49, no. 4, pp. 701-717, Jan. 2018.

[24] Qian, M. S., Jiang, B., and Xu, D. Z., "Fault tolerant control scheme design for the formation control system of unmanned aerial vehicles," Proc. Inst. Mech. Eng. Part I J. Syst. Control Eng., vol. 227, no. 8, pp. 626-634, Aug. 2013.

[25] Qian, M. S., Jiang, B., and Liu, H. T., "Dynamic surface active fault tolerant control design for the attitude control systems of UAV with actuator fault,” Int. J. Control Autom. Syst., vol. 14, no. 3, pp. 723-732, Jun. 2016.

[26] Azizi, S. M. and Khorasani, K., "A hierarchical architecture for cooperative actuator fault estimation and accommodation of formation flying satellites in deep space," IEEE Trans. Aerosp. Electron. Syst., vol. 48, no. 2, pp. 1428-1450, Apr. 2012.

[27] Lan, J. L. and Patton, R. J., "A new strategy for integration of fault estimation within fault-tolerant control," Automatica, vol. 69, pp. 48-59, Jul. 2016.

[28] Lan, J. L. and Patton, R. J., "Integrated fault estimation and fault-tolerant control for uncertain Lipschitz nonlinear systems," Int. J. Robust Nonlinear Control, vol. 27, pp. 761-780, 2017.

[29] Panagi, P. and Polycarpou, M. M., "A coordinated communication scheme for distributed fault tolerant control," IEEE Trans. Ind. Inf., vol. 9, no. 1, pp. 386-393, Feb. 2013.

[30] Zhang, K., Jiang, B., Shi, P., and Cocquempot, V., Observer-based fault estimation techniques, Cham, Switzerland: Springer, 2017.

[31] Ye, D., Chen, M. M., and Li, K., "Observer-based distributed adaptive fault-tolerant containment control of multi-agent systems with general linear dynamics," ISA Trans., vol. 71, no. 1, pp. 32-39, Nov. 2017.

[32] Chen, S., Ho, D. W. C., Li, L. L., and Liu, M., "Fault-tolerant consensus of multi-agent system with distributed adaptive protocol," IEEE Trans. Cybern., vol. 45, no. 10, pp. 2142-2155, Oct. 2015.

[33] Mei, S. W. and Liu, K. Z., Modern robust control theory and application, Beijing, China: Tsinghua University Press, 2003.

[34] Saber, R. O. and Murray, R. M., "Consensus problems in networks of agents with switching topology and time-delays," IEEE Trans. Autom. Control, vol. 49, no. 9, pp. 1520-1533, Sep. 2004. 


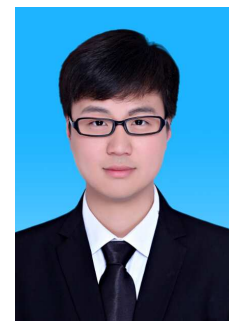

Chun Liu received the B.S. degree in automation and the M.S. degree in control theory and control engineering from the Nanjing University of Aeronautics and Astronautics, Nanjing, China, in 2013 and 2016, respectively, where he is currently pursuing the Ph.D. degree in control theory and control engineering from the Nanjing University of Aeronautics and Astronautics, Nanjing, China.

His current research interests include fault diagnosis and fault tolerant control for multi-agent systems and their applications.

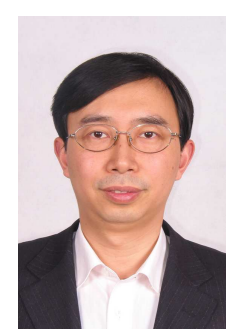

Bin Jiang (SM'05) received the Ph.D. degree in automatic control from Northeastern University, Shenyang, China, in 1995.

He had ever been a Post-Doctoral Fellow, a Research Fellow, an Invited Professor, and a Visiting Professor in Singapore, France, USA and Canada, respectively. He is currently Chair Professor of Cheung Kong Scholar Program with the Ministry of Education and the Vice President of Nanjing University of Aeronautics and Astronautics, Nanjing, China. He has authored eight books and over 200 referred international journal papers and conference papers. His current research interests include intelligent fault diagnosis and fault tolerant control and their applications to helicopters, satellites and high-speed trains.

Dr. Jiang was a recipient of the Second Class Prize of National Natural Science Award of China in 2018. He currently serves as an Associate Editor or an Editorial Board Member for a number of journals, such as the IEEE Transactions on Control Systems Technology, International Journal of Control, Automation and Systems, Journal of Astronautics, Control and Decision, and Systems Engineering and Electronics Technologies. He is a Chair of Control Systems Chapter in IEEE Nanjing Section, a member of IFAC Technical Committee on Fault Detection, Supervision, and Safety of Technical Processes. He has been a Principle Investigator on several projects of National Natural Science Foundation of China.

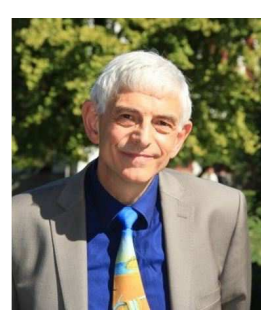

Ron J. Patton (LF'10) received the B.Eng., M.Eng., and Ph.D. degrees in electrical and electronic engineering and control systems from the University of Sheffield, Sheffield, U.K., in 1971, 1974, and 1980, respectively.

He is currently the Chair of Control and Intelligent Systems Engineering, Hull University, Hull, U.K. He has made a substantial contribution in the field of modeling and design of robust methods for fault detection and isolation and fault tolerant control (FTC) in dynamic systems as the author of 376 papers, including 138 journal papers and six books. His research interests include robust, multiple-model and decentralized control strategies for FTC systems and he has a growing interest in FTC methods for renewable energy. He is the Senior Member of AIAA and the Fellow of the Institute of Measurement and Control.

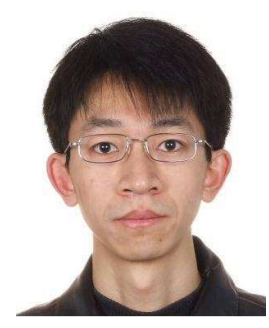

Ke Zhang (SM'17) received the Ph.D. degree in control theory and engineering from the Nanjing University of Aeronautics and Astronautics, Nanjing, China, in 2012.

$\mathrm{He}$ is currently an Associate Professor with the Nanjing University of Aeronautics and Astronautics. His research interests include fault diagnosis and fault tolerant control for dynamical systems and their applications. 